DISTRIBUTION SHEET

\begin{tabular}{lll}
\hline To: & From: & Date: \\
DISTRIBUTION & EAST SYSTEMS ENGINEERING & SEPTEMBER 29, 1994 \\
\hline
\end{tabular}

Project Title/Work Order:

TANK FARMS ESSENTIAL DRAWING PLAN - SD-WM-PC-002

EDT No.: 100799

ECN No.: $N / A$

\begin{tabular}{|c|c|c|c|c|}
\hline Name & MSIN & $\begin{array}{c}\text { with } \\
\text { At tachment }\end{array}$ & $\begin{array}{c}\text { EDT/ECN \& } \\
\text { Comment }\end{array}$ & $\begin{array}{c}\text { EDT/ECN } \\
\text { Only }\end{array}$ \\
\hline
\end{tabular}

A. T. ALSTAD

T. G. ARISS

P. L. BROWN

P. A. CLARK

W. D. COLEMAN (3)

R. A. DODD

L. E. FOX

J. E. GEARY

D. S. HARING

D. G. HERT

C. M. TOWNE (2)

R. E. LARSON

J. J. BADDEN

B. D. BIDDLE

W. E. MEEUWSEN

N. J. MILLIKEN

M. P. MARROT

R. J. NICKLAS (2)

S. H. RIFAEY (2)

D. G. SHEPARD

B. E. SALAZAR (5)

M. S. TIFFANY

G. R. TARDIFF

K. A. WHITE

R. W. REED (2)

O.S.T.I. (2)

CENTRAL FILES (2)

STATION 3

\begin{tabular}{ll} 
R2-70 & $X$ \\
S5-08 & $X$ \\
S2-10 & $X$ \\
R3-47 & $X$ \\
S2-01 & $X$ \\
R2-70 & $X$ \\
R3-47 & $X$ \\
S5-14 & $X$ \\
R1-43 & $X$ \\
R2-88 & $X$ \\
S2-02 & $X$ \\
S2-02 & $X$ \\
S5-01 & $X$ \\
S5-14 & $X$ \\
R2-70 & $X$ \\
H4-63 & $X$ \\
B4-54 & $X$ \\
R1-43 & $X$ \\
S2-45 & $X$ \\
R2-88 & $X$ \\
R2-70 & $X$ \\
R2-70 & $X$ \\
R2-70 & $X$ \\
R2-70 & $X$ \\
R1-51 & $X$ \\
L8-07 & $X$ \\
L8-04 & $X$ \\
S2-10 & $X$ \\
\hline
\end{tabular}

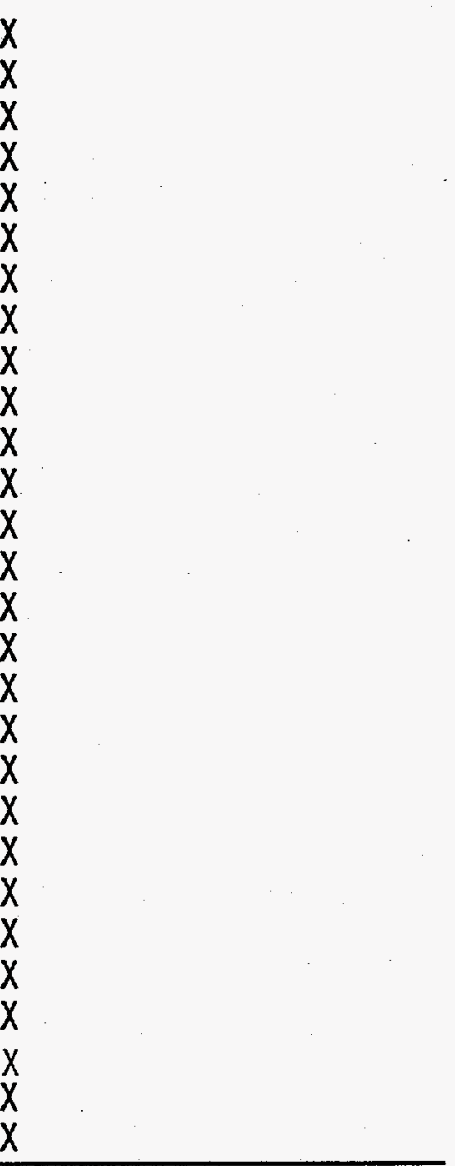




\section{RELEASE AUTHORIZATION}

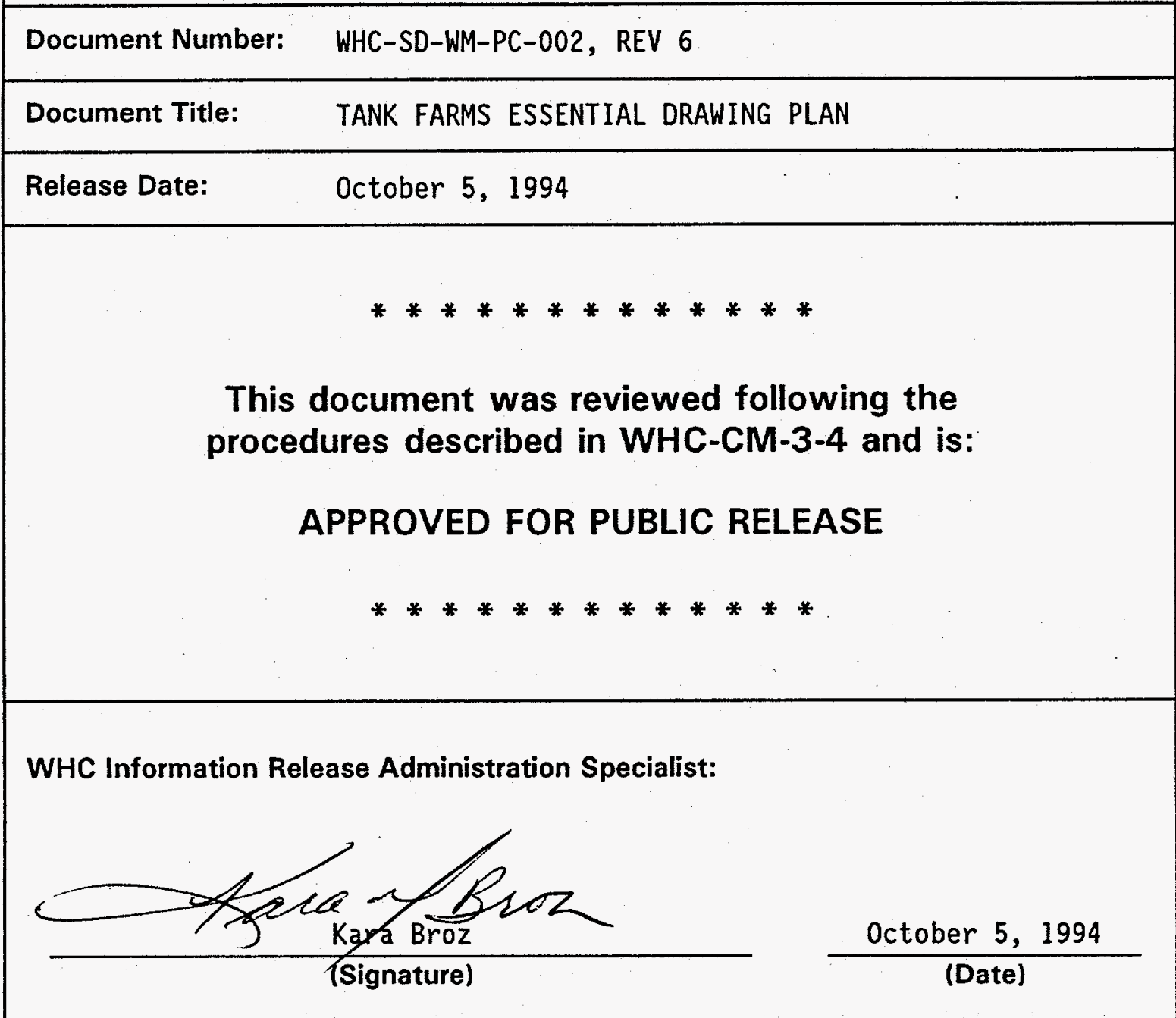




\section{DISCLAIMER}

Portions of this document may be illegible in electronic image products. Images are produced from the best available original document. 
2. Title

TANK FARMS ESSENTIAL DRAWING PLAN

5. Key Words

TANK FARMS ESSENTIAL \& SUPPORT DRAWING PLAN

APPROVED FOR PUBLIC RELEASE
3. Number

WHC-SD-WM-PC-002

6. Author

4. Rev No. 6

Name: Ben

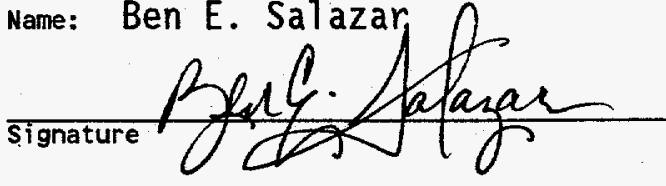

Organization/Charge code 7CF10/N1F6B

7. Abstract

This supporting document provides a detailed list of the essential and support drawings selected for the tank farm(s) and associated facilities.

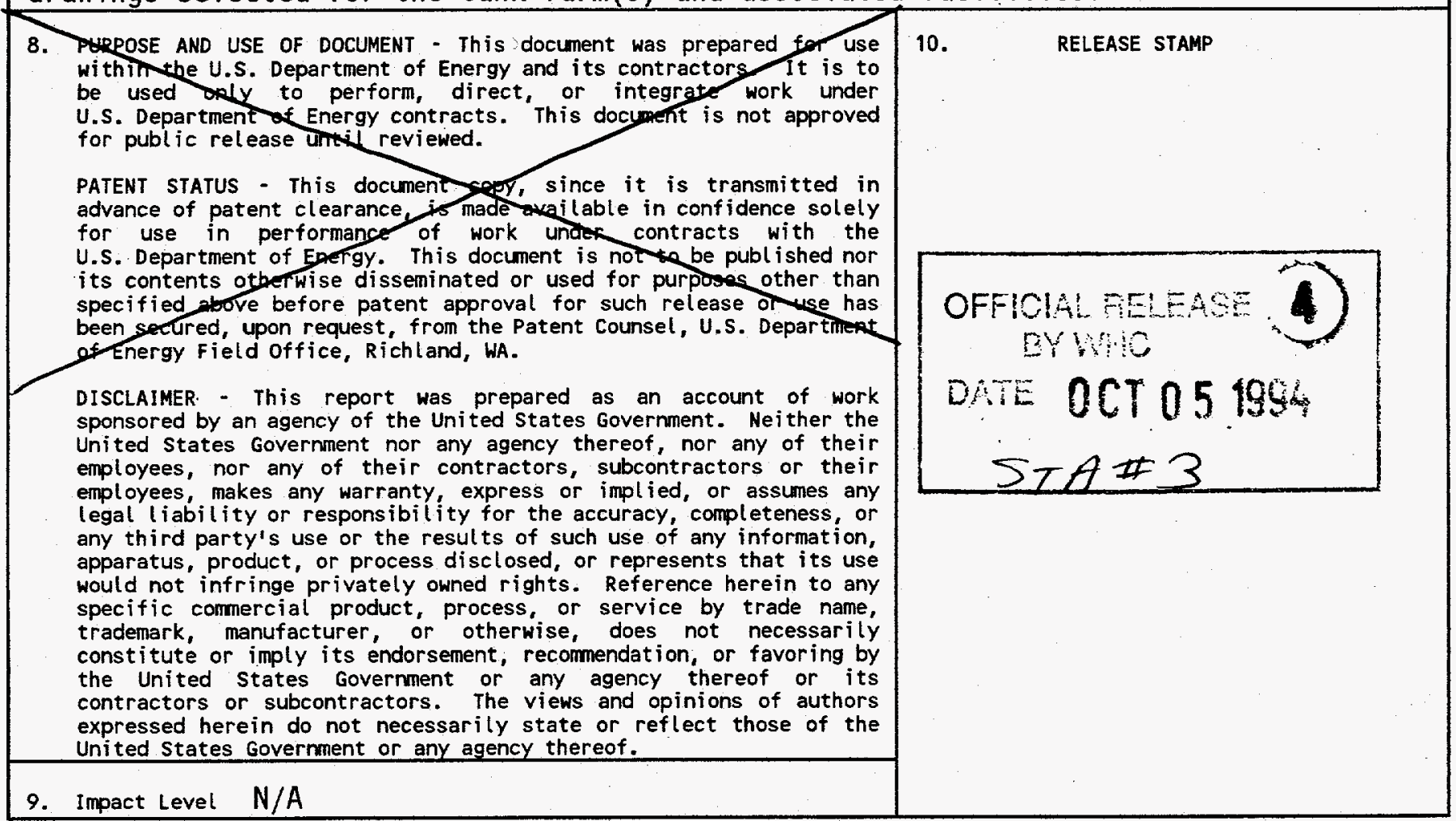




\section{RECORD OF REVISION}

(2) Title

TANK FARMS ESSENTIAL DRAWING PLAN

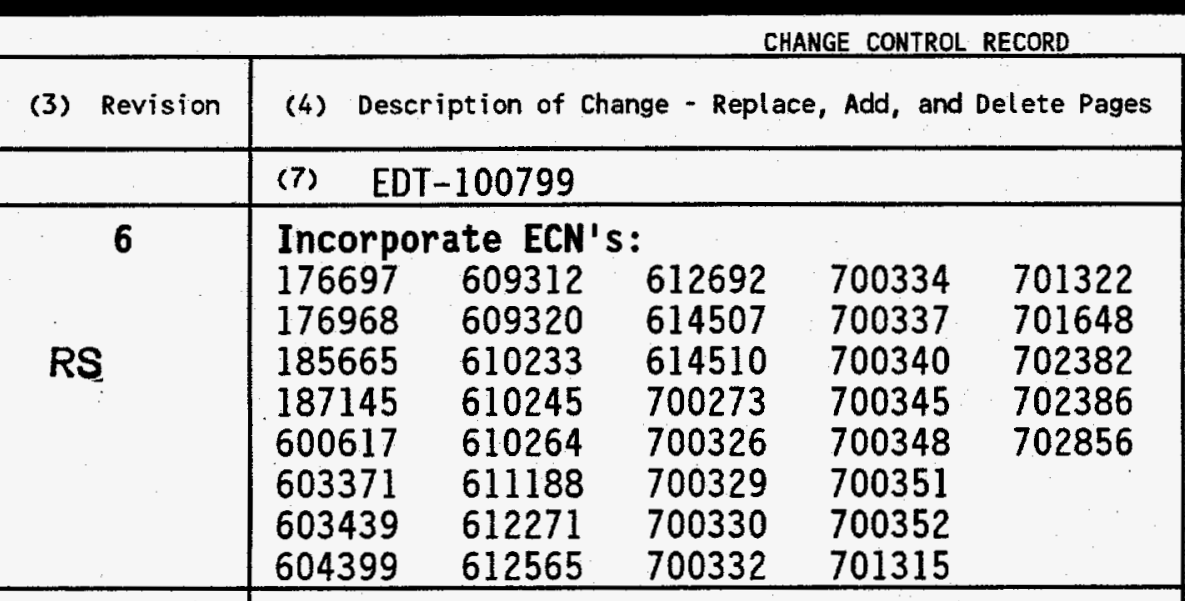

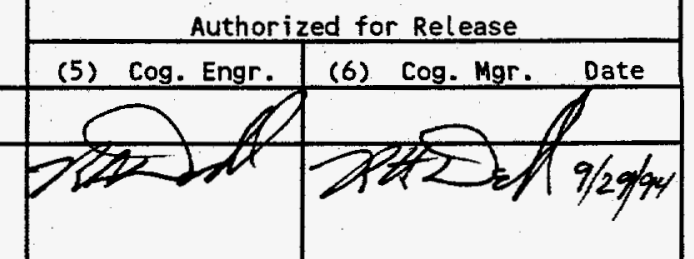

\begin{tabular}{l|l}
\hline & \\
\hline &
\end{tabular}

\begin{tabular}{|l|l|}
\hline & \\
\hline & \\
\hline & \\
\hline & $\cdots$
\end{tabular}




\subsection{PURPOSE}

The purpose of this document is to delineate the process of identification, categorization, and/or classification of Tank Farm Facilities "essential" drawings. This document provides a detailed list of the essential drawings selected for the Tank Farm Facilities. The essential/support drawings are identified by drawing number and title.

\subsection{DEFINITIONS}

2.1 Essential Drawings. Are those engineering drawings identified by the facility staff as necessary to directly support the safe operation of the facility. (WHC-CM-6-1, Glossary)

2.2 Support Drawings. Are those engineering drawings identified by the facility staff that further describe the design details of equipment, components, or systems shown on the essential drawings or are frequently used by the support staff. (WHC-CM-6-1, Glossary)

2.3 General Drawings. Are those engineering drawings identified by the facility staff as historical in nature, or not frequently used in the operation or maintenance of the facility and are not identified as either essential or support drawings. (WHC-CM-6-1, Glossary)

\subsection{DRAWING SELECTION CRITERIA}

Essential and Support drawings identified in this document are deemed essential or supporting as defined in WHC-CM-6-1, Standard Engineering Practices, Section EP-1.3, "Preparation of Engineering Drawings". These drawings are classified as essential or supporting, but still fall under Section EP-2.2, "Engineering Document Change Control", as specified they will be prepared, revised, and maintained. All other Tank Farm Facility drawings not identified in this document will be considered General Drawings and are prepared, revised, and maintained as defined and specified in Section EP-1.3 and Section EP-2.2.

\subsection{REVISION}

The drawing list and engineering file database are required to be updated when new, modified, or deleted safety systems and/or equipment are identified on the essential or support drawing 1ist. Design Services shall identify the vellums as essential or support drawings during change incorporation. 


\section{ESSENTIAL DRAWING LIST}

\section{1-AY, 241-AZ \& MISCELLANEOUS A-FARM FACILITY ESSENTIAL DRAWINGS}

A-FARM MISCELLANEOUS: $(241-A-271, A-401, A-702, A-701, A-08, A-151$, TK-A$417, A-A$ VALVE PIT, A-B VALVE PIT \& AX-A VALVE PIT)

\section{DRAWING}

$\mathrm{H}-2-36877 / 1-2$

$\mathrm{H}-2-44730 / 1$

$\mathrm{H}-2-44744 / 1$

$\mathrm{H}-2-44744 / 2$

$\mathrm{H}-2-44744 / 3$

$\mathrm{H}-2-55086 / 1-4$

H-2-56142/3-4

$\mathrm{H}-2-56146 / 1$

$\mathrm{H}-2-56146 / 2$

$\mathrm{H}-2-57746 / 1$

$\mathrm{H}-2-57752 / 1$

$\mathrm{H}-2-57755 / 1-2$

$\mathrm{H}-2-62894 / 1$

$\mathrm{H}-2-62895 / 1-3$

$\mathrm{H}-2-68323 / 1$

$\mathrm{H}-2-68335 / 1-7$

$\mathrm{H}-2-69165 / 1$

$\mathrm{H}-2-71781 / 3$

$\mathrm{H}-2-73749 / 1$

$\mathrm{H}-2-75959 / 1$

$\mathrm{H}-2-90925 / 1$

$\mathrm{H}-2-90935 / 1$

$\mathrm{H}-2-92516 / 4$

$\mathrm{H}-2-93169 / 1$

$\mathrm{H}-2-93169 / 2$

$\mathrm{H}-2-93169 / 3$

$\mathrm{H}-2-93206 / 2$

$\mathrm{H}-2-93206 / 4$

$\mathrm{H}-2-93206 / 5$

H-2-94719/1-2

$\mathrm{H}-2-94719 / 3$

$\mathrm{H}-2-94719 / 4$

$\mathrm{H}-2-94719 / 5$

$\mathrm{H}-2-94769 / 1-2$

H-2-99389/1-4

\section{TITLE}

ELECTRICAL, FIRE ALARM PLANS \& DIAGRAMS

ELECTRICAL, COMPRESSOR BLDG. CONNECTIONS \& DETAILS

PANEL 7, ANNUNCIATOR ARRANGEMENT \& ELEMENTARY DIAGRAM

PANEL 7, ANNUNCIATOR LEGEND \& ELEMENTARY DIAGRAM

PANEL 7 , ANNUNCIATOR ARRANGEMENT \& WIRING DIAGRAM

ENG. FLOW DIAGRAMS, OUTSIDE WASTE LINES OF PUREX FACILITY

INSTRUMENT ENGINEERING FLOW DIAGRAM

CONTACT CONDENSER, INSTALLATION INSTR. WIRING DIAGRAM ELEC.

CONTACT CONDENSER, INSTALLATION INSTR. WIRING DIAGRAM ELEC.

ENGINEERING FLOW DIAGRAM

MCC-A4 BLDG. 241-791, EMERG. COOLING WATER, ONE-LINE \& ELEM.

ELECTRICAL WIRING DIAGRAMS

ELEC., COMPRESSOR BLDG., $300 \mathrm{KW}$ EMERGENCY POWER GENERATOR INSTALLATION

INSTR. ENG. FLOW DIA., BLDG. 241-702, VENT SYSTEM EXPANSION

ELECTRICAL ONE-LINE DIAGRAM, INSTR.

INSTRUMENT ENGINEERING FLOW DIAGRAMS, 241-AZ TANK FARM

HVAC, VENT FLOW DIAGRAMS, 241-A-TANK FARM

ELECTRICAL, SUBSTATION "A" ARRGT. \& CONNECTION DIAGRAMS

ENGINEERING FLOW DIAGRAM, 241-A \& AX TANK FARMS

INSTR. /ELECTRICAL, ENGINEERING FLOW DIAGRAM

ENGINEERING FLOW DIAGRAM \& HYDRAULIC DIAGRAM

DRAWING LIST, ELEC./INSTR., ENGINEERING FLOW DIAGRAM

FILTER BLDG., STACK MONITOR INSTR., 296-A-17

FILTER BLDG., VENT FAN CONTROL DIAGRAM

FILTER BLDG., VENT FAN CONTROL DIAGRAM

4000 CFM EXHAUSTER, CONTROL DIAGRAM

ELECTRICAL, VALVE PIT 241-A-A JUMPER GRAPHIC SCHEMATIC

ELECTRICAL, GRAPHIC WIRING DIAGRAM, VALVE PIT 241-A-A JUMPER

ELECTRICAL, GRAPHIC WIRING DIAGRAM, VALVE PIT 241-A-A JUMPER 4000 CFM PORTABLE EXAUSTER, MISC. ELEC. DETAILS

4000 CFM EXHAUSTER, STACK SAMPLER WIRING DIAGRAM

4000 CFM EXHAUSTER, INSTR. ENGINEERING FLOW DIAGRAM

4000 CFM PORTABLE EXHAUSTER, INTERCONNECTION DIAGRAM

COMPRESSOR BLDG., IEFD

TANK FARMS 241-A, AX \& AY, ONE-LINE DIAGRAMS 
A-FARM MISCELLANEOUS: (241-A-271, A-401, A-702, A-08, A-151, TK-A-417, A-A VALVE (CON'T) PIT, A-B VALVE PIT \& AX-A VALVE PIT)

$\mathrm{H}-2-74600 / 3$

$\mathrm{H}-2-74600 / 4$

$\mathrm{H}-2-76575 / 1$

$\mathrm{H}-2-76578 / 1$

$\mathrm{H}-2-76578 / 2$

$\mathrm{H}-2-90393 / 1$

$\mathrm{H}-2-92964 / 1-2$

$\mathrm{H}-2-93292 / 2$

$\mathrm{H}-2-94850 / 1$

\section{A, AX-TANK FARMS}

$\mathrm{H}-2-35769 / 2$

$\mathrm{H}-2-36877 / \mathrm{l}-2$

$\mathrm{H}-2-38385 / 1$

$\mathrm{H}-2-69162 / 1$

$\mathrm{H}-2-69174 / 1$

$\mathrm{H}-2-73737 / 1$

$\mathrm{H}-2-73738 / 1$

$\mathrm{H}-2-73749 / 1$

$\mathrm{H}-2-74858 / 2$

$\mathrm{H}-2-85300 / 1$

$\mathrm{H}-2-85300 / 10$

$\mathrm{H}-2-90370 / 2$

$\mathrm{H}-2-91107 / 1-2$

$\mathrm{H}-2-94769 / 2$

$\mathrm{H}-2-97518 / 1$

$\mathrm{H}-2-97667 / 1$

$\mathrm{H}-2-97667 / 2$

$\mathrm{H}-2-99389 / 1-4$
AY \& AZ TANK FARM, LEAK DETECTION SYSTEM, ELECTRICAL DIAGRAM AY \& AZ TANK FARM, PUMP CONTROL DIAGRAM

ELECTRICAL ENGINEERING FLOW DIAGRAM

ELECTRICAL ELEMENTARY DIAGRAM

ELECTRICAL ELEMENTARY DIAGRAM

ELECTRICAL ENGINEERING FLOW DIAGRAMS

ANNULUS VENTILATION CONTROL DIAGRAM \& EQUIPMENT SCHEDULES 241-AZ, ANNULUS EXH. STACK MONITOR, FLOW DIAGRAM \& ELEC. MODIFICATION, ONE-LINE DIAGRAM, PANELBOARD B

ELECTRICAL DIAGRAMS, HEATED MOBILE EXHAUST UNIT

ELECTRICAL, FIRE ALARM PLANS \& DIAGRAMS

INSTRUMENTATION, ELEVATION \& CONNECTION DIAGRAMS

ELECTRICAL, LEAK DETECTION \& MISCELLANEOUS DIAGRAMS

INSTRUMENTATION, RADIATION MONITOR, ARRANGEMENT \& DETAILS

ENGINEERING FLOW DIAGRAM, SALT WELL SYSTEM

ENGINEERING FLOW DIAGRAM, SALT WELL SYSTEM

ENGINEERING FLOW DIAGRAM, 241-A \& 241-AX TANK FARMS

296-P-17, STACK MONITOR FLOW DIAGRAM

PANELBOARD SCHEDULE INDEX

ELECTRICAL PANEL SCHEDULE

EFD, WASTE TRANSFER FACILITIES PHASE III \& IV

ELECTRICAL ONE-LINE \& ELEMENTARY DIAGRAMS

COMPRESSOR BUILDING, IEFD

ELECTRICAL, RADIO FIRE ALARM PLAN \& DIAGRAMS

P-17, MOBILE EXHAUST UNIT, ELECTRICAL PLANS \& DETAILS

P-17, MOBILE EXHAUST UNIT, CONTROL PANEL, ELEC. SCHEMATIC

TANK FARMS 241-A, AX \& AY ONE-LINE DIAGRAMS

\section{B, BX, AND BY-TANK FARMS}

$\mathrm{H}-2-34306 / 1$

$\mathrm{H}-2-34692 / 1$

$\mathrm{H}-2-38376 / 1$

$\mathrm{H}-2-38385 / 1$

$\mathrm{H}-2-70880 / 1-2$

$\mathrm{H}-2-73600 / 1-2$

$\mathrm{H}-2-73601 / 1$

$\mathrm{H}-2-73605 / 1-2$

$\mathrm{H}-2-73606 / 1$

$\mathrm{H}-2-73819 / 2$
ELECTRICAL CONTROL \& POWER - ITS \#2

ONE-LINE DIAGRAM

ELECTRICAL ELEMENTARY DIAGRAM

INSTRUMENTATION, ELEVATIONS \& CONNECTION DIAGRAMS

ENGINEERING FLOW DIAGRAMS, SALT WELL SYSTEM

ELECTRICAL ONE-LINE DIAGRAMS

ELECTRICAL JET PUMP, CONTROL WIRING DIAGRAM

ELECTRICAL ANNUNCIATOR, ELEMENTARY WIRING DIAGRAMS

ELECTRICAL HEAT TRACE \& LEAK DETECTION, ELEMENTARY DIAGRAMS

ELECTRICAL ONE-LINE \& ELEM. DIAGRAM, SYSTEM NO. 2 


\section{C-FARM AND 244-CR}

$\mathrm{H}-2-33944 / 1-2$

$\mathrm{H}-2-35769 / 2$

$\mathrm{H}-2-36670 / 1-2$

$\mathrm{H}-2-36671 / 1$

$\mathrm{H}-2-38385 / 1$

$\mathrm{H}-2-62061 / 1$

$\mathrm{H}-2-73799 / 1$

$\mathrm{H}-2-73833 / 1$

$\mathrm{H}-2-73948 / 1$

$\mathrm{H}-2-73950 / 1$

$\mathrm{H}-2-73970 / 1$

$\mathrm{H}-2-73971 / 1$

$\mathrm{H}-2-91116 / 1$

$\mathrm{H}-2-91116 / 2$

H-2-92519/1-4

$\mathrm{H}-2-95267 / 2$

$\mathrm{H}-2-97519 / 1$

\section{S-TANK FARM}

$\mathrm{H}-2-35047 / 1$

$\mathrm{H}-2-35047 / 2$

$\mathrm{H}-2-70613 / 1-3$

$\mathrm{H}-2-71854 / 1$

$\mathrm{H}-2-73737 / 1$

$\mathrm{H}-2-73738 / 1$

$\mathrm{H}-2-91107 / 2$

\section{SX-TANK FARM}

$\mathrm{H}-2-35047 / 1$

$\mathrm{H}-2-35047 / 2$

$\mathrm{H}-2-35834 / 1$

$\mathrm{H}-2-35849 / 1$

$\mathrm{H}-2-36874 / 1$

$\mathrm{H}-2-36875 / 1$

$\mathrm{H}-2-38934 / 1$

$\mathrm{H}-2-70613 / 1-3$

$\mathrm{H}-2-71854 / 1$

$\mathrm{H}-2-74913 / 3$

$\mathrm{H}-2-91107 / 2$
IEFD, VAULT TANKS INSTRUMENTATION

ELECTRICAL DIAGRAMS, ELECTRICAL HEATED MOBILE EXHAUST

FIRE PROTECTION, SPRINKLER SYSTEM

ELECTRICAL, FIRE PROTECTION SYSTEM

INSTRUMENTATION, ELEVATION \& CONNECTION DIAGRAMS

EFD, 244-CR VAULT MODIFICATIONS

EFD, SYSTEM NO. 4

ELECTRICAL ELEMENTARY DIAGRAM, SYSTEM NO. 4

ELECTRICAL ONE-LINE DIAGRAM, 241-C-T FARMS

LEAK DETECTION, ELEMENTARY DIAGRAM

ENGINEERING FLOW DIAGRAM, SALT WELL SYSTEM

ENGINEERING FLOW DIAGRAM, SALT WELL SYSTEM

ELECTRICAL ONE-LINE, JET PUMP, ELEMENTARY DIAGRAM

ELECTRICAL ONE-LINE \& ELEMENTARY DIAGRAMS

STACK MONITOR INSTALLATION, 296-C-5

296-P-16, STACK MONITOR FLOW DIAGRAM

ELECTRICAL, RADIO FIRE ALARM PLAN \& DIAGRAMS
$S \&$ SX TANK FARM, ONE-LINE DIAGRAMS

$S$ \& SX TANK FARM, PANEL SCHEDULES \& MCC ARRANGEMENTS

ELECTRICAL ONE-LINE DIAGRAMS

AREA RADIATION ADDITION TO MASTER SHUTDOWN CIRCUIT

ENGINEERING FLOW DIAGRAM, SALT WELL SYSTEM

ENGINEERING FLOW DIAGRAM, SALT WELL SYSTEM

ELECTRICAL ONE-LINE \& ELEMENTARY DIAGRAMS

\section{I, TX, AND TY-TANK FARMS}

$\mathrm{H}-2-2813 / 1$

$\mathrm{H}-2-36047 / 1$

$\mathrm{H}-2-36282 / 1$

$\mathrm{H}-2-38617 / 1$

$\mathrm{H}-2-73948 / 1$
$S$ \& SX TANK FARM, ONE-LINE DIAGRAMS

$S$ \& SX TANK FARM, PANEL SCHEDULES \& MCC ARRANGEMENTS

VENTILATION AIR FLOW DIAGRAM, TANK FARM 241-SX

ENGINEERING FLOW DIAGRAM, TANK FARM 241-SX

ELECTRICAL PLANS, ONE-LINE DIAGRAM, SCHEDULES \& FIRE ALARM

ELECTRICAL, FIRE ALARM PLAN \& CONTROL DIAGRAM

ELECTRICAL PLANS, ONE-LINE DIAGRAM \& FIRE ALARM

ELECTRICAL ONE-LINE DIAGRAMS

AREA RADIATION ADDITION TO MASTER SHUTDOWN SCHEDULE

296-S-15, STACK MONITOR INSTALLATION

ELECTRICAL ONE-LINE DIAGRAM 


\section{I, TX, AND TY-TANK FARMS (CON'T)}

$\mathrm{H}-2-73950 / 1$

$\mathrm{H}-2-73970 / 1$

$\mathrm{H}-2-73971 / 1$

$\mathrm{H}-2-91116 / 1$

$\mathrm{H}-2-91116 / 2$

\section{U-TANK FARM}

$\mathrm{H}-2-36874 / 1$

$\mathrm{H}-2-38934 / 1$

$\mathrm{H}-2-73682 / 2$

$\mathrm{H}-2-73703 / 1-2$

$\mathrm{H}-2-73704 / 1$

\section{4-AR VAULT}

$\mathrm{H}-2-36878 / 1$

$\mathrm{H}-2-63939 / 1$

$\mathrm{H}-2-63940 / 1$

$\mathrm{H}-2-70696 / 2$

$\mathrm{H}-2-70697 / 2-3$

$\mathrm{H}-2-76717 / 1$

$\mathrm{H}-2-76717 / 2$

$\mathrm{H}-2-76717 / 3$

$\mathrm{H}-2-76717 / 4$

$\mathrm{H}-2-76717 / 5$

$\mathrm{H}-2-76718 / 4$

$\mathrm{H}-2-79949 / 1-14$

$\mathrm{H}-2-91191 / 3$

$\mathrm{H}-2-92484 / 4$

$\mathrm{H}-2-94016 / 1$

$\mathrm{H}-2-99111 / 1$

\section{2-S EVAPORATOR}

$\mathrm{H}-2-46424 / 1-3$

\section{2-A EVAPORATOR}

$\mathrm{H}-2-69294 / 1-2$

$\mathrm{H}-2-69330 / 1-2$

$\mathrm{H}-2-69332 / 1$

$\mathrm{H}-2-79865 / 2$

$\mathrm{H}-2-79865 / 3$

$\mathrm{H}-2-79865 / 1$

$\mathrm{H}-2-79865 / 5$

$\mathrm{H}-2-79865 / 4$

$\mathrm{H}-2-79865 / 6$
LEAK DETECTION, ELEMENTARY DIAGRAM

ENGINEERING FLOW DIAGRAM, SALT WELL SYSTEM

ENGINEERING FLOW DIAGRAM, SALT WELL SYSTEM

ELECTRICAL ONE-LINE, JET PUMP, CONTROL ELEMENTARY DIAGRAM

ELECTRICAL ONE-LINE \& ELEMENTARY DIAGRAMS

ELECTRICAL PLANS, ONE-LINE DIAGRAM \& FIRE ALARM

ELECTRICAL PLANS, ONE-LINE DIAGRAM \& FIRE ALARM

ELECTRICAL, JET PUMP, CONTROL ONE-LINE \& ELEMENTARY DIAGRAMS

ENGINEERING FLOW DIAGRAMS, SALT WELL SYSTEM

ENGINEERING FLOW DIAGRAM, SALT WELL SYSTEM

ELEC., FIRE ALARM SYSTEM \& DIESEL GENERATOR RELOCATION

ENGINEERING FLOW DIAGRAM, 244-AR VAULT

ENGINEERING FLOW DIAGRAM, CONTAINMENT VAULT

ELECTRICAL DETAILS \& ELEMENTARY DIAGRAMS

HVAC, PLAN, SECTIONS \& DETAILS

HVAC, INSTRUMENT AIRFLOW \& CONTROL DIAGRAM

HVAC, INSTRUMENT AIRFLOW \& CONTROL DIAGRAM

HVAC, INSTRUMENT AIRFLOW \& CONTROL DIAGRAM

HVAC, INSTRUMENT AIRFLOW \& CONTROL DIAGRAM

HVAC, INSTRUMENT AIRFLOW \& CONTROL DIAGRAM

INSTRUMENT PLAN \& DETAILS

IEFD, SLUDGE REMOVAL \& WASTE TRANSFER VAULT

296-A-13, STACK MONITOR FLOW DIAGRAM

VESSEL VENT STACK MONITOR, FLOW DIAGRAM

ENG. FLOW DIAGRAM, WASTE TRANSFER FACILITIES, PHASE $V$

IEFD, COOLING WATER SAMPLER MONITOR UPGRADE

\section{ELECTRICAL ONE-LINE DIAGRAM}

HEATING \& VENTILATION, AIR FLOW \& CONTROL DIAGRAMS ENGINEERING FLOW DIAGRAMS, AIR SAMPLING/CAM ALARMS FLOW DIAGRAM, SC RETENTION BASINS \& 207-A BUILDING FIRE PROTECTION, SPRINKLER SYSTEM, 2ND FLOOR PLAN FIRE PROTECTION, SPRINKLER SYSTEM, COND. RM \& PLATFORM PLANS FIRE PROTECTION, SPRINKLER SYSTEM, IST FLOOR PLAN FIRE PROTECTION, SPRINKLER SYSTEM, IST \& 2ND FLOOR DETAILS FIRE PROTECTION, SPRINKLER SYSTEM, IST FLOOR DETAILS FIRE PROTECTION, SPRINKLER SYSTEM, CONDENSER ROOM DETAILS 


\section{2-A EVAPORATOR (CON'T)}

$\mathrm{H}-2-85087 / 1$

$\mathrm{H}-2-85087 / 2$

$\mathrm{H}-2-85087 / 3$

$\mathrm{H}-2-85322 / 1-7$

$\mathrm{H}-2-92393 / 1-4$

$\mathrm{H}-2-92505 / 3-4$

$\mathrm{H}-2-98986 / 1-3$

H-2-98988/1-2

$\mathrm{H}-2-98989 / 1$

$\mathrm{H}-2-98990 / 1-2$

$\mathrm{H}-2-98991 / 1-2$

$\mathrm{H}-2-98992 / 1$

$\mathrm{H}-2-98993 / 1$

$\mathrm{H}-2-98994 / 1$

$\mathrm{H}-2-98995 / 1-2$

$\mathrm{H}-2-98996 / 1$

$\mathrm{H}-2-98997 / 1$

$\mathrm{H}-2-98998 / 1$

$\mathrm{H}-2-98999 / 1$

$\mathrm{H}-2-99000 / 1$

$\mathrm{H}-2-99001 / 1-2$

$\mathrm{H}-2-99002 / 1$

$\mathrm{H}-2-99003 / 1$

$\mathrm{H}-2-99059 / 1-13$

$\mathrm{H}-2-99085 / 1-24$

$\mathrm{H}-2-99949 / 1-24$

$\mathrm{H}-2-99949 / 28-54$

$\mathrm{H}-2-100096 / 1-3$
FIRE ALARM PLAN, SCHEDULE \& DETAIL

FIRE ALARM PLAN, DETAILS \& LEGEND

FIRE ALARM DIAGRAM \& SCHEDULES

PANELBOARD SCHEDULES

STACK MONITOR, FLOW DIAGRAMS, 296-A-21

296-A-22, VESSEL VENT STACK MONITOR WIRING, FLOW DIAGRAM

P\&ID, LEGEND \& INTERLOCK SCHEDULE

P\&ID, EVAPORATOR RECIRC. SYSTEM

P\&ID, SLURRY SYSTEM

P\&ID, PROCESS CONDENSATE SYSTEM

P\&ID, RAW WATER SYSTEM

P\&ID, STEAM SYSTEM

P\&ID, STEAM CONDENSATE SYSTEM

P\&ID, USED RAW WATER SYSTEM

P\&ID, DRAIN SYSTEM

P\&ID, DECONTAMINATION SYSTEM

P\&ID, EFFLUENT SYSTEM

P\&ID, VESSEL VENT SYSTEM

P\&ID, VACUUM CONDENSER SYSTEM

P\&ID, ANTI-FOAM SYSTEM

P\&ID, PROCESS \& INSTRUMENT AIR SYSTEM

P\&ID, JET GANG VALVE SYSTEM

P\&ID, FILTERED RAW WATER SYSTEM

INSTRUMENT INTERCONNECTION DIAGRAMS

ELECTRICAL ELEMENTARY DIAGRAMS

MCS, LOGIC DIAGRAMS

MCS, LOGIC DIAGRAMS

ELECTRICAL ONE-LINE DIAGRAMS

\section{2-AL LERF}

$\mathrm{H}-2-79601 / 2$

$\mathrm{H}-2-79601 / 3$

$\mathrm{H}-2-79602 / 2$

$\mathrm{H}-2-79649 / 1-2$

$\mathrm{H}-2-79649 / 3-6$

$\mathrm{H}-2-79653 / 1-2$

$\mathrm{H}-2-79659 / 1$

$\mathrm{H}-2-79663 / 1$

$\mathrm{H}-2-79667 / 1-2$

$\mathrm{H}-2-79667 / 3$

$\mathrm{H}-2-79668 / 2-4$

$\mathrm{H}-2-79668 / 5$

$P$ \& ID EVAPORATOR BLDG. \& XCY PIPING

$P$ \& ID 242-A EVAP. RETENTION BASINS

$P$ \& ID 242-A EVAP. RETENTION BASINS

INSTR. LEACHATE LEVEL DETECTION \& WIRING RETENTION BASIN

INSTR. BASIN LEVEL DETECTION \& WIRING RETENTION BASIN

INSTR. UHF/PLC WIRING DIAGRAM

INSTRUMENTATION LOGIC DIAGRAM

ELECTRICAL ONE-LINE DIAGRAM \& PANEL SCHEDULE

ELECTRICAL RETENTION BASIN AREA PLANS \& DETAILS

ELECTRICAL RETENTION BASIN AREA SUBSTATION PLANS \& DETAILS

ELECTRICAL ELEMENTARY DIAGRAM

ELECTRICAL ELEMENTARY DIAGRAM \& PANEL SCHEDULES

\section{1-G TANK FARMS GENERAL}

$\mathrm{H}-2-36535 / 1$

$\mathrm{H}-2-36535 / 2$

$\mathrm{H}-2-36535 / 3$

$\mathrm{H}-2-36535 / 4$
SANITARY \& RAW WATER MAP, 200-EAST AREA

SANITARY WATER VALVE IDENTIFICATION, 200-EAST AREA

RAW WATER VALVE IDENTIFICATION, 200-EAST AREA

FIRE HYDRANT IDENTIFICATION, 200-EAST AREA 
241-G TANK FARMS GENERAL (CON'T)

$\mathrm{H}-2-36535 / 5$

$\mathrm{H}-2-36535 / 6-10$

$\mathrm{H}-2-73450 / 1-4$

$\mathrm{H}-2-85327 / 1-5$

$\mathrm{H}-2-90718 / 1-6$

$\mathrm{H}-2-95331 / \mathrm{ALL}$

$\mathrm{H}-2-95365 / \mathrm{ALL}$

$\mathrm{H}-14-020000 / 1-2$
SANITARY \& RAW WATER MAP DETAILS, 200-EAST AREA

SANITARY \& RAW WATER MAP, 200-EAST AREA

PIPING, ISOLATION DETAILS, PIPE \& RISER CLOSURES

INDEX \& PANELBOARD SCHEDULES

PIPING, AIR FILTER INSTALLATION, ATMOSPHERIC BREATHING LIQUID LEVEL REEL ARRANGEMENT

LIQUID LEVEL PLUMMET

$\mathrm{H}-14$ SYMBOL LEGEND

\section{4-AR}

$\mathrm{H}-2-70694 / 2$

$\mathrm{H}-2-70694 / 3$

$\mathrm{H}-2-70695 / 1$

$\mathrm{H}-2-70695 / 2$

$\mathrm{H}-2-70695 / 7$

$\mathrm{H}-2-70696 / 1-2$

$\mathrm{H}-2-70697 / 1-3$

$\mathrm{H}-2-70699 / 1-2$

$\mathrm{H}-2-70703 / 1-3$

$\mathrm{H}-2-71558 / 1$

$\mathrm{H}-2-79852 / 1-4$

$\mathrm{H}-2-85195 / 1$

$\mathrm{H}-2-92187 / 1$

$\mathrm{H}-2-92187 / 4$

$\mathrm{H}-2-95302 / 1$

$\mathrm{H}-2-99036 / 1$

H-2-99070/2-3
ELECTRICAL LIGHTING \& FIRE ALARM PLAN, IST FLOOR

ELECTRICAL LIGHTING \& FIRE ALARM PLAN, 2ND FLOOR

ELECTRICAL DIAGRAM \& PANEL SCHEDULES

ELECTRICAL WIRING DIAGRAM

ELECTRICAL WIRING DIAGRAM

HVAC, SCHEDULES, DIAGRAMS \& DETAILS

HVAC, PLAN, SECTIONS \& DETAILS

FIRE PROTECTION SPRINKLER SYSTEM, PLANS \& SECTIONS

ENGINEERING FLOW DIAGRAMS

ELEC./INSTR., SUBSTATION "A" CONNECTION DIAGRAM

ELECTRICAL POWER PLAN, 1ST FLOOR

ELECTRICAL ONE-LINE DIAGRAM

AIR MONITOR \& CONTROL INSTALLATION

AIR MONITOR \& CONTROL WIRING DIAGRAM

COMPRESSOR AFTER COOLER INSTALLATION

PIPING, FUEL OIL STORAGE TANK

ELEC. GEN. RELOCATION PLAN, DETAILS \& DIAGRAMS

\section{1-AY, 241-AZ \& MISCELLANEOUS A-FARM FACILITIES}

$\mathrm{H}-2-36877 / 1-2$

$\mathrm{H}-2-64347 / 1-2$

$\mathrm{H}-2-64370 / 1-2$

$\mathrm{H}-2-64400 / 1-5$

$\mathrm{H}-2-64400 / 6-9$

$\mathrm{H}-2-70796 / 1-2$

$\mathrm{H}-2-70797 / 1-2$

$\mathrm{H}-2-90256 / 1$

$\mathrm{H}-2-90370 / 2$

$\mathrm{H}-2-90393 / 1$

$\mathrm{H}-2-92066 / 1$

$\mathrm{H}-2-92482 / 1$

$\mathrm{H}-2-93292 / 1$

$\mathrm{H}-2-93373 / 2$
ELECTRICAL FIRE ALARM PLANS \& DIAGRAMS

MCC-AY1, BLDG. 241-AY-801, INSTR. HOUSE PLAN \& DETAIL 241-AY-801, REAR PANEL WIRING \& ELEM. DIAGRAMS

ENGINEERING FLOW DIAGRAM

ENGINEERING FLOW DIAGRAM, 241-AY TANK FARM

ELECTRICAL DETAILS \& ELEMENTARY DIAGRAM

ELECTRICAL HEAT TRACE, DIAGRAMS \& DETAILS

ELECTRICAL DETAILS

EFD, WASTE TRANSFER FACILITIES, PHASE III \& IV

ELECTRICAL ENG INEERING FLOW DIAGRAMS

VENTILATION EQUIPMENT, SCHEDULES \& CONTROL DIAGRAM MODIFICATIONS

ANNULUS EXHAUST SYSTEM, MODIFICATION DIAGRAM

241-AZ ANNULUS STACK MONITOR, INSTRUMENTATION

241-AY-101 ANNULUS EXHAUST STACK MONITOR, FLOW DIAGRAM 
241-AY, 241-AZ \& MISCELLANEOUS A-FARM FACILITIES (CON'T)

$\mathrm{H}-2-93374 / 2$

$\mathrm{H}-2-94016 / 1$

$\mathrm{H}-2-94028 / 1$

$\mathrm{H}-2-94029 / 1-3$

$\mathrm{H}-2-94769 / 2$

$\mathrm{H}-2-99389 / 1-4$

\section{4-U FARM}

$\mathrm{H}-2-73682 / 2$

$\mathrm{H}-2-73798 / 1-2$

$\mathrm{H}-2-73809 / 1$

$\mathrm{H}-2-73820 / 1$

$\mathrm{H}-2-73823 / 1$

$\mathrm{H}-2-73823 / 2$

$\mathrm{H}-2-73826 / 1-2$

$\mathrm{H}-2-73838 / 2$

$\mathrm{H}-2-73839 / 1-2$

$\mathrm{H}-2-97499 / 1$

\section{4-BX FARM}

H-2-73797/1-4

$\mathrm{H}-2-73809 / 1$

$\mathrm{H}-2-73819 / 1$

$\mathrm{H}-2-73819 / 2$

$\mathrm{H}-2-73822 / 1-2$

$\mathrm{H}-2-73825 / 1-2$

$\mathrm{H}-2-73838 / 1-2$

$\mathrm{H}-2-73839 / 1-2$

$\mathrm{H}-2-97537 / 1$

\section{1-AN TANK FARM}

$\mathrm{H}-2-71560 / 1$

$\mathrm{H}-2-71561 / 1$

$\mathrm{H}-2-71927 / 1-3$

$\mathrm{H}-2-71931 / 1-2$

$\mathrm{H}-2-71933 / 1$

H-2-71944/1

$\mathrm{H}-2-71945 / 1$

H-2-71946/1

$\mathrm{H}-2-71959 / 1-3$

$\mathrm{H}-2-71968 / 1$

$\mathrm{H}-2-71978 / 1-2$

$\mathrm{H}-2-71979 / 1$

$\mathrm{H}-2-71980 / 1$

$\mathrm{H}-2-71981 / 1$

$\mathrm{H}-2-71982 / 1$
241-AY-101 ANNULUS EXH. STACK MONITOR, FLOW DIAGRAM \& ELEC.

ENG. FLOW DIAGRAM, WASTE TRANSFER FACILITIES, PHASE V

ELECTRICAL ENGINEERING FLOW DIAGRAM

ELECTRICAL ELEMENTARY DIAGRAM

COMPRESSOR BUILDING, IEFD

TANK FARMS 241-A, AX \& AY, ONE-LINE DIAGRAMS

ELECTRICAL JET PUMP CONTROL, ONE-LINE \& ELEMENTARY DIAGRAM IEFD, SYSTEM NO. 3

INSTRUMENT EFFLUENT MONITORING

SYSTEM NO. 3, ELECTRICAL LINE

ELEMENTARY DIAGRAM, SYSTEM NO. 3

ELEC. LEAK DET., HEAT TRACE \& ANN. ELEM. DIAG., SYSTEM NO. 3 ELECTRICAL POWER DISTRIBUTION HVAC, AIRFLOW \& CONTROL DIAGRAM \& SCHEDULE

HVAC, PLANS, SECTIONS \& DETAILS

FIRE ALARM

INSTRUMENT ENGINEERING FLOW DIAGRAM (IEFD)

INSTRUMENT EFFLUENT MONITORING

SYSTEM NO. 2, ELECTRICAL ONE-LINE DIAGRAM

ELECTRICAL ONE-LINE \& ELEM. DIAG., SYSTEM NO. 2

ELECTRICAL DIAGRAMS, SYSTEM NO. 2

ELECTRICAL POWER DISTRIBUTION

HVAC, AIRFLOW \& CONTROL DIAGRAMS \& SCHEDULE

HVAC, PLANS, SECTIONS \& DETAILS

FIRE ALARM

ELEC./INSTR., SUBSTATION "C" CONNECTION DIAGRAM

ELEC./INSTR., SUBSTATION " $C$ " CONNECTION DIAGRAM

ELECTRICAL POWER \& CONTROL

ELECTRICAL LEAK DETECTION, ELEMENTARY DIAGRAMS

ELECTRICAL, INSTRUMENT, ELEMENTARY DIAGRAM

IEFD, TANKS 241-AN-101, $102 \& 103$

INSTR. ENG. FLOW DIAGRAM, TANKS 241-AN-104 THRU 107

INSTR., TANKS 241-AN-101 THRU 107, RADIATION MONITORING

SCHEMATICS

INSTRUMENT ANNUNCIATOR, ELEMENTARY DIAGRAMS

INSTRUMENT FLOW \& RADIATION MONITORING SYSTEM

ENGINEERING FLOW DIAGRAMS, 241-AN TANK FARM

ENGINEERING FLOW DIAGRAM, TANK 241-AN-101

ENGINEERING FLOW DIAGRAM, TANK 241-AN-102

ENGINEERING FLOW DIAGRAM, TANK 241-AN-103

ENGINEERING FLOW DIAGRAM, TANK 241-AN-104 


\section{1-AN TANK FARM (CON'T)}

$\mathrm{H}-2-71983 / 1$

$\mathrm{H}-2-71984 / 1$

$\mathrm{H}-2-72008 / 1$

$\mathrm{H}-2-72037 / 1$

$\mathrm{H}-2-73800 / 1$

$\mathrm{H}-2-79892 / 1$

$\mathrm{H}-2-85259 / 7$

$\mathrm{H}-2-85259 / 12$

$\mathrm{H}-2-85301 / 5-6$

$\mathrm{H}-2-97521 / 1$

$\mathrm{H}-2-97524 / 1$

$\mathrm{H}-2-99310 / 5$

$\mathrm{H}-2-99310 / 6$

$\mathrm{H}-14-010501 / 1$

$\mathrm{H}-14-010501 / 2$

$\mathrm{H}-14-020501 / 1$

$\mathrm{H}-14-020601 / 1$

H-14-030001/1-2

$\mathrm{H}-14-030001 / 5$

H-14-030001/6-12

H-14-030003/1-2

$\mathrm{H}-14-030003 / 5$

H-14-030003/6-12

H-14-030003/13-20

$\mathrm{H}-14-030201 / 1$

$\mathrm{H}-14-030301 / 1$

\section{1-SY TANK FARM}

H-2-37735/1-3

$\mathrm{H}-2-37744 / 1$

$\mathrm{H}-2-37757 / 1-2$

$\mathrm{H}-2-37761 / 1$

H-2-46424/4-6

$\mathrm{H}-2-46788 / 1$

$\mathrm{H}-2-76532 / 1$

$\mathrm{H}-2-76544 / 1$

$\mathrm{H}-2-79556 / 1$

$\mathrm{H}-2-85104 / 1$

$\mathrm{H}-2-85256 / 1$

$\mathrm{H}-2-85314 / 1-5$

$\mathrm{H}-2-85314 / 6$

$\mathrm{H}-2-93427 / 3$

$\mathrm{H}-2-97479 / 1-2$
ENGINEERING FLOW DIAGRAM, TANK 241-AN-105

ENGINEERING FLOW DIAGRAM, TANK 241-AN-106

PIT \& PIT COVER PAINTING DIAGRAM

ENGINEERING FLOW DIAGRAM, TANK 241-AN-107

ENGINEERING FLOW DIAGRAM, SYSTEM NO. 5

IEFD, COMPRESSED AIR

ELECTRICAL-CAUSTIC ADD: PROJECT-PART. ONE-LINE DIAG. \& DETAIL

ELECTRICAL-CAUSTIC ADDITION PROJECT ELEMENTARY DIAGRAMS

ELECTRICAL, CAUSTIC ADDITION PROJECT PANELBOARD

ELECTRICAL, RADIO FIRE ALARM PLAN \& DIAGRAM

ELECTRICAL, RADIO FIRE ALARM PLAN \& DIAGRAM

296-A-29, VESSEL VENT EXHAUST STACK SAMPLER/MONITOR

296-A-30, ANNULUS EXHAUST STACK SAMPLER/MONITOR

TANK PENITRATIONS

TANK 107 PENITRATIONS

ANNULUS TANK INSTRUMENTATION

PRIMARY TANK INSTRUMENTATION

MCC-101/102 ELECTRICAL ONE-LINE

PANELBOARD SCHEDULE INDEX

PANELBOARD SCHEDULES

MCC ELECTRICAL ONE-LINE

PANELBOARD SCHEDULE INDEX

PANELBOARD SCHEDULES

PANELBOARD SCHEDULES

ANNULUS TANK VENTILATION POWER \& CONTROL

SERVICE AIR/GLYCOL SYSTEM ELECTRICAL DIAGRAM

ELECTRICAL ELEMENTARY DIAGRAMS

VENTILATION AIR FLOW \& CONTROL DIAGRAM

INSTRUMENTATION, ENGINEERING FLOW DIAGRAMS, 241-SY TANKS. 101, $102 \& 103$

INSTRUMENTATION, ANNUNCIATOR ELEMENTARY DIAGRAM

ELECTRICAL ONE-LINE DIAGRAM

ELECTRICAL/INSTRUMENTATION, ENG. FLOW DIAGRAM \& DRAWING LIST

ENGINEERING FLOW DIAGRAM, 241-SY TANK FARM

JUMPER ASSEMBLY, 241-SY-03A, A-(PUMP DISCHARGE)-G

241-SY-101, RISER EQUIPMENT ARRANGEMENT

241-SY-103, RISER EQUIPMENT ARRANGEMENT

TANK 102, RISER EQUIPMENT ARRANGEMENT

PANELBOARD SCHEDULES

PANELBOARD SCHEDULE BACKUP GENERATOR

296-P-22 \& 296-P-23, STACK MONITOR, FLOW DIAGRAM

ELECTRICAL RADIO FIRE ALARM PLAN \& DIAGRAM 
244-TX DCRT

$\mathrm{H}-2-73796 / 1-5$

$\mathrm{H}-2-73818 / 1-2$

$\mathrm{H}-2-73838 / 2$

$\mathrm{H}-2-73839 / 1-2$

$\mathrm{H}-2-97547 / 1$
ENGINEERING FLOW DIAGRAM, SYSTEM NO. 1

ELECTRICAL ONE-LINE \& ELEMENTARY DIAGRAMS, SYSTEM NO. 1

HVAC, AIRFLOW \& CONTROL DIAGRAM \& SCHEDULE

HVAC, PLANS, SECTIONS \& DETAILS

ELECTRICAL, RADIO FIRE ALARM PLAN \& DIAGRAM

\title{
244-S DCRT
}

$\mathrm{H}-2-71085 / 1-2$

$\mathrm{H}-2-71091 / 1-2$

$\mathrm{H}-2-71092 / 1$

$\mathrm{H}-2-97500 / 1$

INSTRUMENTATION, ENGINEERING FLOW DIAGRAMS

ELECTRICAL CONTROL \& ELEMENTARY DIAGRAMS

ELECTRICAL DIAGRAM \& DETAILS \& PANEL BOARD SCHEDULES

ELECTRICAL, RADIO FIRE ALARM PLAN \& DIAGRAM

\section{1-EW-151 VENT STATION}

$\mathrm{H}-2-76532 / 1$

$\mathrm{H}-2-76554 / 1$

$\mathrm{H}-2-91825 / 2$

ENGINEERING FLOW DIAGRAM, 241-SY TANK FARM

ELECTRICAL ONE-LINE \& ELEMENTARY DIAGRAM

SERIAL ALARM TRANSMITTER, PCB ASSEMBLY

\section{1-TX-152-154 DIVERSION BOXES}

$241-\mathrm{TX}-152$

$\mathrm{H}-2-34965 / 1-3$

LEAK DETECTOR ASSEMBLY, TYP. DETAILS \& ELEMENTARY DIAGRAMS

\section{1-AP TANK FARM}

\author{
$\mathrm{H}-2-76506 / 1-2$ \\ $\mathrm{H}-2-76515 / 1-4$ \\ $\mathrm{H}-2-90476 / 1-13$ \\ $\mathrm{H}-2-90487 / 1-2$ \\ $\mathrm{H}-2-90488 / 1-6$ \\ $\mathrm{H}-2-90514 / 1-2$ \\ $\mathrm{H}-2-97526 / 1$ \\ $\mathrm{H}-2-99134 / 1$ \\ $\mathrm{H}-14-030003 / 3$
}

IEFD, 102-AP

ELECTRICAL ELEMENTARY DIAGRAMS

ELECTRICAL ELEMENTARY DIAGRAMS

IEFD, TANKS 101 THRU 108

IEFD, MISC. DIAGRAMS

HVAC, FLOW \& CONTROL DIAGRAMS

ELECTRICAL, RADIO FIRE ALARM PLAN \& DIAGRAM

EFD, COMPRESSOR COOLING WATER

ELECTRICAL ONE-LINE DIAGRAM

\section{1-ER DIVERSION BOXES \& CATCH TANKS}

$\mathrm{H}-2-38223 / 1$

$\mathrm{H}-2-90370 / 1-2$

$\mathrm{H}-2-90398 / 1$

\section{4-A LIFT STATION}

$\mathrm{H}-2-38209 / 1$

$\mathrm{H}-2-38211 / 1$

$\mathrm{H}-2-38217 / 1$

$\mathrm{H}-2-90399 / 1-2$

$\mathrm{H}-2-90502 / 4$

$\mathrm{H}-2-95298 / 3$
EFD, WASTE TRANSFER SYSTEM

EFD, WASTE TRANSFER FACILITIES, PHASE III \& IV

EFD, ELECTRICAL

ELECTRICAL ONE-LINE DIAGRAM

ELECTRICAL CONTROL DIAGRAM

INSTRUMENT ENGINEERING FLOW DIAGRAM (IEFD)

ELECTRICAL ELEMENTARY DIAGRAMS

ELEC. / INSTR. , ELEMENTARY DIAGRAM

296-A-25, STACK FLOW DIAGRAM 
241-A-350 CATCH TANK

$\mathrm{H}-2-70322 / 1$

$\mathrm{H}-2-70325 / 1-4$

$\mathrm{H}-2-70352 / 2$

$\mathrm{H}-2-70387 / 2$

$\mathrm{H}-2-92348 / 2$

\section{1-AW TANK FARM}

$\mathrm{H}-2-70322 / 1$

$\mathrm{H}-2-70324 / 1$

$\mathrm{H}-2-70324 / 2-3$

$\mathrm{H}-2-70324 / 4$

$\mathrm{H}-2-70324 / 5$

$\mathrm{H}-2-70325 / 1-4$

$\mathrm{H}-2-70327 / 1-2$

$\mathrm{H}-2-70327 / 3$

$\mathrm{H}-2-70329 / 1-2$

$\mathrm{H}-2-70331 / 1$

$\mathrm{H}-2-70336 / 1$

$\mathrm{H}-2-70348 / 1-2$

$\mathrm{H}-2-70349 / 1$

$\mathrm{H}-2-70350 / 1$

$\mathrm{H}-2-70351 / 1$

H-2-70362/1-2

$\mathrm{H}-2-74896 / 2$

$\mathrm{H}-2-74897 / 3$

$\mathrm{H}-2-79872 / 1-2$

H-2-85303/1-10

$\mathrm{H}-2-90276 / 1$

$\mathrm{H}-2-90905 / 1$

$\mathrm{H}-2-90927 / 1$

$\mathrm{H}-2-95219 / 1$

$\mathrm{H}-2-97521 / 1$

$\mathrm{H}-2-97524 / 1$

$\mathrm{H}-14-010502 / 1$

$\mathrm{H}-14-020502 / 1$

$\mathrm{H}-14-020602 / 1$

H-14-030302/1
ELECTRICAL ONE-LINE DIAGRAM

ELECTRICAL ELEMENTARY DIAGRAMS

INSTR., DRAINAGE LIFT STATION

IEFD, DRAINAGE LIFT STATION

PUMP PIT, POWER \& FLOW METER UPGRADE
ELECTRICAL ONE-LINE DIAGRAM

ELECTRICAL PLANS, PANELBOARD SCHEDULE \& DETAILS

ELECTRICAL PLANS, PANELBOARD SCHED. \& CONTROL RELAY PANEL.

ELECTRICAL PLANS, PANELBOARD SCHED., RELAY \& TERMINAL CAB.

ELECTRICAL PLANS, PANELBOARD SCHED. \& DETAILS

ELECTRICAL POWER \& CONTROL

ELECTRICAL, HEAT TRACE, ELEMENTARY DIAGRAMS

ELECTRICAL, HEAT TRACE, ELEM. DIAG. \& CONTROL PANEL

ELECTRICAL, LEAK DETECTION, ELEMENTARY DIAGRAMS

ELECTRICAL, INSTRUMENT, ELEMENTARY DIAGRAM

HVAC, FLOW \& CONTROL DIAGRAM

IEFD, TANK-241-AW-101, $103 \& 104$

IEFD, TANK-241-AW-102

IEFD, TANK-241-AW-105 \& 106

INSTR., TANKS 241-AW-101 THRU 106, RADIATION MONITORING SCHEMATICS

INSTRUMENT, ANNUNCIATOR, ELEMENTARY DIAGRAMS

296-A-27, STACK MONITOR, FLOW DIAGRAM

296-A-28, ANNULUS TANK, EXHAUST STACK MONITOR INSTALLATION

IEFD, COMPRESSED AIR

PANELBOARD SCHEDULES

ELECTRICAL ELEMENTARY DIAGRAM

HVAC, FLOW CONTROL DIAGRAM

ELECTRICAL ONE-LINE DIAGRAM \& DETAILS

INSTRUMENT, AIR COMPRESSOR PACKAGE, ELEMENTARY DIAGRAM

ELECTRICAL, RADIO FIRE ALARM PLAN \& DIAGRAM

ELECTRICAL, RADIO FIRE ALARM PLAN \& DIAGRAM

TANK PENITRATIONS

ANNULUS TANK INSTRUMENTATION

PRIMARY TANK INSTRUMENTATION

SERVICE AIR/INSTRUMENT AIR ELECTRICAL ELEMENTARY DIAGRAM

\section{COMPUTER AUTOMATED SURVEILLANCE SYSTEM (CASS)}

$\mathrm{H}-2-38933 / 1$

$\mathrm{H}-2-38936 / 1$

$\mathrm{H}-2-38937 / 1$

$\mathrm{H}-2-38938 / 1$

$\mathrm{H}-2-38939 / 1$

$\mathrm{H}-2-38940 / 1$

$\mathrm{H}-2-38941 / 1$
INSTRUMENTATION, SURVEILLANCE, DATA COMMUNICATION SYSTEM, 200E/200W

ELECTRICAL, SURVEILLANCE SYSTEM, DATA LINES, 2503W

ELECTRICAL, SURVEILLANCE SYSTEM, DATA LINES, 2503W

ELECTRICAL, SURVEILLANCE SYSTEM, DATA LINES, 2503W

ELECTRICAL, SURVEILLANCE SYSTEM, DATA LINES, 2503E/2503W

ELECTRICAL, SURVEILLANCE SYSTEM, DATA LINES, 2503E/W

ELECTRICAL, SURVEILLANCE SYSTEM, DATA LINES, $2503 E$ 
COMPUTER AUTOMATED SURVEILLANCE SYSTEM (CASS) (CON'T)

$H-2-38942 / 1$
$H-2-38943 / 1$
$H-2-69156 / 1$
$H-2-71555 / 1$
$H-2-71563 / 1$
$H-2-71564 / 1$
$H-2-71568 / 1$
$H-2-71781 / 3-4$
$H-2-71782 / 2$
$H-2-71783 / 2$
$H-2-71785 / 2-3$
$H-2-71789 / 2$
$H-2-71791 / 1$
$H-2-71791 / 2$
$H-2-71793 / 1$
$H-2-71794 / 1-2$
$H-2-71796 / 1$
$H-2-71797 / 1$
$H-2-71797 / 2$
$H-2-71797 / 3$
$H-2-71797 / 4$
$H-2-71799 / 1$
$H-2-71800 / 1$
$H-2-71800 / 2$
$H-2-71800 / 3$
$H-2-71801 / 1-2$
$H-2-71801 / 3$
$H-2-71801 / 4$
$H-2-71802 / 3$
$H-2-71802 / 1$
$H-2-71802 / 2$
$H-2-71804 / 1$
$H-2-71804 / 2$
$H-2-71804 / 3$
$H-2-71805 / 1$
$H-2-71805 / 2$
$H-71556 / 1$
$H-71616 / 1$
$H$
$H$

ELECTRICAL, SURVEILLANCE SYSTEM, DATA LINES, 2503W ELECTRICAL, SURVEILLANCE SYSTEM, DATA LINES, $2503 E$ ELECTRICAL POWER \& INSTRUMENT PLANS, 241-A \& 241-AX ELEC./INSTR., SUBSTATION "A" CONN. DIAG., 242-A ELEC./INSTR., SUBSTATION "S" CONNECTION DIAGRAM, 242-S ELEC./INSTR., SUBSTATION "S" CONN. DIAGRAM, 241-SY/271-BLDG. ELEC./INSTR., SUB. "U" ALARM CONN. DIAG., 241-U \& 241-U27 ELECTRICAL, SUBSTATION "A" ARRANGEMENT \& CONNECTION DIAGRAM, 241-A

ELECTRICAL, SUBSTATION "B" ARRANGEMENT \& CONNECTION DIAGRAM, 241-B

ELEC. , SUBSTATION "C" ARRANGEMENT CONN. DIAGRAM, 241-CR ELECTRICAL, SUBSTATION "T" ARRANGEMENT \& CONNECTION DIAGRAM, 241-T, 241-TX \& 242-TC

ELEC., CASS CENTRAL SUBSTATION, ARRANGEMENT \& CONNECTION DIAGRAM, 200-GENERAL

CASS, ALARM INTERFACE MOTHER BOARD LAYOUT, 241-GENERAL

CASS, ALARM INTERFACE MOTHER BOARD, DRILL \& TRIM, 241-GEN.

CASS, ALARM INTERFACE ALARM RECEIVER MODULE, 241-GENERAL

CASS, CABLE DETAILS, 200-GENERAL

CASS, FRONT PANEL MODULE, 200-GENERAL

PANEL TERMINATOR \& CONTROL/ALARM BOARD, 200-GENERAL

PANEL TERMINATOR CONTROL/ALARM BOARD LAYOUT, 200-GENERAL

PANEL TERMINATOR BOARD FOR FRONT PANEL DRAWER, DRILL \& TRIM CONTROL/ALARM BOARD FOR FRONT PANEL DRAWER, DRILL \& TRIM, 200-GENERAL

BRACKET ASSEMBLY, 200-GENERAL

TRANS/RCVR BOARD FOR MICROPAC 80 SCHEMATIC, 241-GENERAL

TRANS/RCVR BOARD FOR MICROPAC 80 ASSEMBLY, 241-GENERAL

TRANS/RCVR BOARD FOR MICROPAC 80 P.C. BOARD FABRICATION, 241-GENERAL

INTERFACE BOARD FOR LIQUID LEVEL GAUGES, SCHEMATIC, 241-GENERAL

INTERFACE BOARD FOR LIQUID LEVEL GAUGES, ASSEMBLY, 241-GENERAL

INTERFACE BOARD FOR LIQUID LEVEL GAUGES, P.C. BOARD FABRICATION, 241-GENERAL

FIC, GAUGE INTERFACE BOARD P.C. FABRICATION, 241-GENERAL FIC, GAUGE INTERFACE BOARD SCHEMATIC, 241-GENERAL FIC, GAUGE INTERFACE BOARD ASSEMBLY, 241-GENERAL

CASS, ALARM LOOP INTERFACE READER SCHEMATIC, 241-GENERAL CASS, ALARM LOOP INTERFACE READER LAYOUT, 241-GENERAL . CASS, ALARM LOOP READER, DRILL \& TRIM, 241-GENERAL CASS, ALARM LOOP INTERFACE TRANSMITTER LAYOUT, 241-GENERAL CASS, ALARM LOOP INTERFACE TRANSMITTER SCHEMATIC, 241-GEN. ELEC./INSTR., SUBSTATION CONNECTION DIAGRAM, 241-AW ELEC./INSTR., SUBSTATION "A" CONNECTION DIAGRAM, 204-AR ELEC./INSTR., LIQUID LEVEL GAUGE CONNECTION DIAGRAM, 200E/W 
COMPUTER AUTOMATED SURVEILLANCE SYSTEM (CASS) (CON'T)

H-2-71617/1

$\mathrm{H}-2-71784 / 2-3$

$\mathrm{H}-2-71786 / 2$

$\mathrm{H}-2-7.1787 / 1$

$\mathrm{H}-2-71790 / 2$

$\mathrm{H}-2-71790 / 1$

$\mathrm{H}-2-71790 / 3$

$\mathrm{H}-2-71792 / 3$

$\mathrm{H}-2-71792 / 1$

$\mathrm{H}-2-71795 / 1$

$\mathrm{H}-2-71798 / 1$

$\mathrm{H}-2-72386 / 1$

$\mathrm{H}-2-72386 / 2$

$\mathrm{H}-2-72387 / 1$

$\mathrm{H}-2-72387 / 2$

$\mathrm{H}-2-76507 / 2$

$\mathrm{H}-2-76508 / 2$

$\mathrm{H}-2-90507 / 1$

$\mathrm{H}-2-90507 / 2$

$\mathrm{H}-2-90507 / 3$

$\mathrm{H}-2-90507 / 4$

$\mathrm{H}-2-90507 / 5$

$\mathrm{H}-2-90507 / 6$

$\mathrm{H}-2-90508 / 1$

$\mathrm{H}-2-91825 / 1$

$\mathrm{H}-2-91826 / 1$

$\mathrm{H}-2-91827 / 1$

$\mathrm{H}-2-91828 / 1$

$\mathrm{H}-2-91829 / 1$

$\mathrm{H}-2-91830 / 1$

$\mathrm{H}-2-98471 / 1$
ELEC./INSTR., LIQUID LEVEL GAUGE CONNECTION DIA. \& DETAILS, 200E/W

ELECTRICAL, SUBSTATION "S" ARRANGEMENT \& CONNECTION DIAG., 241-SX

ELECTRICAL, SUBSTATION "U" ARRANGEMENT \& CONNECTION DIAG., 241-U

CASS, INTERFACE VENT STATION LEAK DETECTOR, 241-EW

CASS, ALARM INTERFACE TRANSMITTER BOARD LAYOUT, 241-GENERAL

CASS, ALARM INTERFACE TRANSMITTER BOARD SCHEMATIC, 241-GEN.

CASS, ALARM INTERFACE TRANS. BOARD, DRILL \& TRIM, 241-GEN.

CASS, ALARM INTERFACE RECEIVER BOARD, DRILL \& TRIM, 241-GEN.

CASS, ALARM INTERFACE RECEIVER BOARD SCHEMATIC, 241-GENERAL

LIQUID LEVEL ALARM INTERFACE INSTALLATION, 241-GENERAL

FRONT PANEL MODULE CABLES, 200-GENERAL

PRINTED WIRING BOARD, CASS, AUDIBLE ALARM GENERATOR, $2750 E$

SCHEMATIC DIAGRAM, CASS, AUDIBLE ALARM GENERATOR, $2750 E$

CASS, AUDIBLE ALARM MODULE, 2750E

CASS, AUDIBLE ALARM MODULE SCHEMATIC, $2750 E$

INSTRUMENT \& PANEL ARRANGEMENT \& DETAILS, 241-AP

/271-BLDG.

INSTR., CASS, ALARM INTERFACE CONNECTION DIAGRAM, 241-AP

INSTR., CASS, ALARM INTERFACE CONNECTION DIAGRAM, 241-AP 1242-A

INSTR., CASS, ALARM INTERFACE CONNECTION DIAGRAM, 241-AP INSTR., CASS, ALARM INTERFACE CONNECTION DIAGRAM, 241-AP /242-A

INSTR., CASS, ALARM INTERFACE CONNECTION DIAGRAM, 242-A

INSTR., CASS, ALARM INTERFACE CONNECTION DIAGRAM, 24I-AP 1242-A

INSTR., CASS, ALARM INTERFACE CONNECTION DIAGRAM, 241-AP

/242-A

INSTR., CASS, SUBSTA. "A" FIC INTERFACE CONN. DIAG., 241-AP /241-AZ

SERIAL ALARM TRANSMITTER, PCB ASSEMBLY, 241-GENERAL

SERIAL ALARM TRANSMITTER, SCHEMATIC DIAGRAM, 241-GENERAL

SERIAL ALARM RECEIVER, PCB ASSEMBLY, 241-GENERAL

SERIAL ALARM RECEIVER, SCHEMATIC DIAGRAM, 241-GENERAL

SERAIL ALARM RECEIVER, MODULE ASSEMBLY, 241-GENERAL

SERIAL ALARM RECEIVER, MODULE DETAILS, 241-GENERAL

INSTR., CASS, ALARM INTERFACE CONNECTION DIAGRAM, 218-E16. 


\section{SUPPORT DRAWING LIST}

\section{1-SY TANK FARM}

\section{DRAWING}

$\mathrm{H}-2-37383 / 1$

$\mathrm{H}-2-37732 / 1-3$

$\mathrm{H}-2-37733 / 1-2$

$\mathrm{H}-2-37734 / 1$

$\mathrm{H}-2-37736 / 1-2$

$\mathrm{H}-2-37745 / 1$

$\mathrm{H}-2-37746 / 1-3$

$\mathrm{H}-2-37747 / 1$

$\mathrm{H}-2-37758 / 1-3$

$\mathrm{H}-2-37759 / 1$

$\mathrm{H}-2-37766 / 1-2$

$\mathrm{H}-2-37767 / 1$

$\mathrm{H}-2-37768 / 1$

$\mathrm{H}-2-37769 / 1$

$\mathrm{H}-2-37770 / 1-2$

$\mathrm{H}-2-37777 / 1-2$

$\mathrm{H}-2-37778 / 1-3$

$\mathrm{H}-2-37779 / 1$

$\mathrm{H}-2-37780 / 1$

$\mathrm{H}-2-37781 / 1$

$\mathrm{H}-2-37782 / 1$

$\mathrm{H}-2-37783 / 1$

$\mathrm{H}-2-37784 / 1$

$\mathrm{H}-2-37785 / 1$

H-2-37787/1-2

$\mathrm{H}-2-37793 / 1-4$

$\mathrm{H}-2-37801 / 1$

$\mathrm{H}-2-37802 / 1$

$\mathrm{H}-2-37803 / 1$

$\mathrm{H}-2-37809 / 1$

$\mathrm{H}-2-37811 / 1$

$\mathrm{H}-2-37812 / 1$

$\mathrm{H}-2-37846 / 1-2$

$\mathrm{H}-2-37847 / 1$

$\mathrm{H}-2-46421 / 2-3$

$\mathrm{H}-2-46785 / 2-3$

\section{TITLE}

ELECTRICAL, LEAK DETECTION ALARM S, SX, SY \& U

ELECTRICAL, SITE PLAN

ELECTRICAL, HEAT TRACE PLANS

ELEC./INSTR., HOUSE PLAN, ONE-LINE DIAGRAM \& PANEL BOARD

ELECTRICAL DETAILS

VENTILATION DUCTWORK PLAN

VENTILATION EQUIPMENT PLAN \& DETAILS

VENTILATION EQUIPMENT SCHEDULES

INSTRUMENTATION, PANEL ARRANGEMENT, INSTRUMENT BUILDING

INSTRUMENTATION, REAR PANEL PIPING, INSTRUMENT BUILDING

INSTRUMENTATION, IN-TANK PRESSURE ASSEMBLIES, PLAN \& DETAILS

ENGINEERING FLOW DIAGRAM, 241-SY TANK 101

ENGINEERING FLOW DIAGRAM, 241-SY TANK 102

ENGINEERING FLOW DIAGRAM, 241-SY TANK 103

ENGINEERING FLOW DIAGRAM, 241-SY TANK FARM MISCELLANEOUS

PIPING PLAN, 241-SY TANK FARM, 242-S BLDG. AREA

PIPING PLAN, 241-SY TANK FARM

JUMPER ARRANGEMENT, VALVE PITS 241-SY-A \& SY-B

PIPING PLAN, VALVE PITS 241-SY-A \& B

PIPING SECTIONS \& DETAILS, VALVE PITS 241-SY-A \& B

JUMPER ARRANGEMENT, PUMP PIT 241-SY-01A, 02A \& 03A

PIPING PLAN \& DETAILS, PUMP PIT 241-SY-01A, 02A \&03A

EQUIPMENT ARRANGEMENT, ANNULUS PUMP PIT, 241-SY-01B, 02B \& O3B, LEAK DETECTION PIT, 241-SY-01C, 02C \& 03C

PIPING PLAN \& DETAILS, ANNULUS PUMP PIT, 241-SY-01B, 02B \& O3B

PIPING PLAN \& DETAILS, LEAK DETECTION PIT, 241-SY-01C, $02 C$ \& 03C

PIPING PLAN \& DETAILS, FLUSH PIT, 241-SY-A \& B

PIPING PLAN, TANK 101

PIPING PLAN, TANK 102

PIPING PLAN, TANK 103

ARRANGEMENT \& DETAILS, DRAIN PIT, 241-SY-02D

JUMPER ARRANGEMENT, FEED PUMP PIT, 241-SY-02E

PIPING PLAN \& DETAILS, FEED PUMP PIT, 241-SY-02E \& FLUSH PIT, 241-SY-02E

INSTRUMENTATION, PRESSURE SWITCH RACK, ARRANGEMENT \& DETAILS

INSTRUMENTATION, ANNUNCIATOR PANEL ARRANGEMENT \& WIRING

FIRE PROTECTION, SPRINKLER SYSTEM, IST \& 2ND FLOOR PLANS

INSTRUMENTATION, EXHAUST UNIT ENCLOSURE ASSEMBLY 
241-SY TANK FARM (CON'T)

H-2-46789/1-2

$\mathrm{H}-2-68503 / 1$

$\mathrm{H}-2-70211 / 1$

$\mathrm{H}-2-71559 / 1$

$\mathrm{H}-2-71784 / 1$

$\mathrm{H}-2-72396 / 1$

$\mathrm{H}-2-92999 / 1-4$

$\mathrm{H}-2-93427 / 4$

$\mathrm{H}-2-93427 / 1$

\section{4-TX DCRT}

$\mathrm{H}-2-73805 / 1-2$

$\mathrm{H}-2-73810 / 1$

$\mathrm{H}-2-73811 / 1$

$\mathrm{H}-2-73812 / 1$

$\mathrm{H}-2-73812 / 2$

$\mathrm{H}-2-73815 / 1$

H-2-73824/1-5

$\mathrm{H}-2-73830 / 1$

$\mathrm{H}-2-73831 / 1$

$\mathrm{H}-2-74001 / 1$

\section{4-S DCRT}

$\mathrm{H}-2-46421 / 2-3$

$\mathrm{H}-2-46784 / 1$

$\mathrm{H}-2-46785 / 1-3$

$\mathrm{H}-2-46786 / 1$

$\mathrm{H}-2-71047 / 1$

$\mathrm{H}-2-71048 / 1$

$\mathrm{H}-2-71050 / 1-2$

$\mathrm{H}-2-71087 / 1-2$

H-2-71094/1

$\mathrm{H}-2-71784 / 1$

$\mathrm{H}-2-73801 / 1$

$\mathrm{H}-2-73841 / 1$

\section{C-FARM \& 244-CR}

$\mathrm{H}-2-95267 / 1$

$\mathrm{H}-2-95267 / 3$
ELECTRICAL/INSTRUMENTATION PLANS, ELEVATIONS, DETAILS \& ELEMENTARY DIAGRAMS

ENGINEERING FLOW DIAGRAM, WASTE TRANSFER, 102-SY \& 241-AX-A ENGINEERING FLOW DIAGRAM, AIR LIFT CIRCULATORS

ELEC. /INSTR., EXHAUSTER ELEMENTARY DIAGRAM

ELECTRICAL, SUBSTATION "S" ARRANGEMENT \& CONN. DIAGRAMS LEAK DETECTION, TANK FARM, ALARM MODIFICATIONS

ANNULUS EXHAUST, RADIATION MONITORS \& RADAR LEVEL GAUGE 296-P-22 \& 296-P-23, STACK MONITOR INSTALLATION WIRING DIAG. 296-P-22 \& 296-P-23, STACK MONITOR INSTALLATION

INSTRUMENTATION, INSTRUMENT BUILDING, ARRANGEMENT \& DETAILS INSTRUMENTATION, ANNULUS UNIT ENCLOSURE ASSEMBLY

INSTRUMENTATION, EXHAUST UNIT ENCLOSURE ASSEMBLY

INSTRUMENTATION-INTERCONNECTION DIAGRAM

INSTRUMENTATION, EXHAUST UNIT ENCL. ASSY., SYSTEM NO. 1

INSTRUMENTATION, ANNUNCIATOR ARRANGEMENT \& WIRING

ELECTRICAL POWER, INSTRUMENT, HEAT TRACE, DISTR. PLAN, SYSTEM NO. 1

ELECTRICAL, INSTRUMENT BUILDING 244-BX/TX, SYSTEM NO. 1 \& 2 ELEC., HEAT TRACE, PANEL ARRANGEMENT, SYSTEM NO. 1, 2 \& 3 INSTRUMENTATION, NEUTRON MONITORING ASSEMBLY \& SCHEMATIC

FIRE PROTECTION, SPRINKLER SYSTEM, IST \& 2ND FLOOR PLANS INSTRUMENTATION, ANNULUS UNIT ENCLOSURE ASSEMBLY

INSTRUMENTATION, EXHAUST UNIT ENCLOSURE ASSEMBLY

ELECTRICAL/INSTRUMENTATION, INTERCONNECTION DIAGRAM \&

ENGINEERING FLOW DIAGRAM

VENTILATION AIR FLOW \& CONTROL DIAGRAM \& SCHEDULES

VENTILATION PLAN \& DETAILS

PIPING, ENGINEERING FLOW. DIAGRAMS

INSTRUMENTATION, INSTRUMENT ENCLOSURE 244-S, ARRANGEMENT \& DETAILS

ELECTRICAL ELEMENTARY DIAGRAM \& DETAILS

ELECTRICAL, SUBSTATION "S" ARRANGEMENT \& CONN. DIAGRAM

ENGINEERING FLOW DIAGRAM, SYSTEM NO. 6

ELEC., MODIFICATION TO 241-S, SYSTEM \& ELEMENTARY DIAGRAM, SYSTEM NO. 6

296-P-16 STACK MONITOR INSTALLATION 296-P-16 STACK MONITOR WIRING DIAGRAM 


\section{1-EW-151 VENT STATION}

H-2-76555/1

\section{1-AP TANK FARM}

$\mathrm{H}-2-76471 / 1$

$\mathrm{H}-2-76473 / 1$

$\mathrm{H}-2-76507 / 1$

$\mathrm{H}-2-76508 / 1$

$\mathrm{H}-2-77451 / 1$

$\mathrm{H}-2-77452 / 1$

$\mathrm{H}-2-90457 / 3-4$

H-2-90500/1-4

$\mathrm{H}-2-90522 / 1$

H-2-90526/1-2

$\mathrm{H}-2-90527 / 1$

$\mathrm{H}-2-90528 / 1$

$\mathrm{H}-2-90529 / 1$

$\mathrm{H}-2-90530 / 1$

$\mathrm{H}-2-90531 / 1$

$\mathrm{H}-2-90532 / 1$

$\mathrm{H}-2-90533 / 1$

$\mathrm{H}-2-90540 / 1$

$\mathrm{H}-2-90541 / 1$

$\mathrm{H}-2-90544 / 1$

$\mathrm{H}-2-90545 / 1-3$

$\mathrm{H}-2-90546 / 1$

H-2-90547/1-2

$\mathrm{H}-2-90548 / 1$

H-2-90551/1-2

$\mathrm{H}-2-90553 / 1$

$\mathrm{H}-2-90554 / 1$

H-2-90555/1

$\mathrm{H}-2-90556 / 1$

$\mathrm{H}-2-90557 / 1$

$\mathrm{H}-2-90558 / 1$

H-2-90559/1

$\mathrm{H}-2-90560 / 1$

$\mathrm{H}-2-90562 / 1$

$\mathrm{H}-2-90563 / 1$

H-2-90564/1

$\mathrm{H}-2-90565 / 1$

$\mathrm{H}-2-90566 / 1$

$\mathrm{H}-2-90567 / 1$

$\mathrm{H}-2-90568 / 1-2$

$\mathrm{H}-2-90569 / 1-2$
ELECTRICAL/INSTRUMENTATION, ELEMENTARY DIAGRAM \& DETAILS

EFD, 102-AP

PIPING PLAN \& SECTION, 241-AP-02D PUMP PIT

INSTRUMENT, PANEL ARRANGEMENTS

INSTRUMENT, CASS, ALARM INTERFACE

PIPING PLAN, MIXER PUMP PIT, 241-AP-07D, 07E, 08D \& 08E

PIPING PLAN, MIXER PUMP PIT, 241-AP-07F \& 08F

STRUCTURAL CENTRAL EXHAUST STATION SECTIONS \& DETAILS

INSTRUMENT-INTERCONNECTION DIAGRAMS

HVAC EQUIPMENT SCHEDULES \& NOTES

EFD, 241-AP MISC.

EFD, DRAIN LINES

EFD, 241-AP TANK 101

EFD, 241-AP TANK 102

EFD, 241-AP TANK 103

EFD, 241-AP TANK 104

EFD, 241-AP TANK 105

EFD, 241-AP TANK 106

EFD, 241-AP TANK 107

EFD, 241-AP TANK 108

PIPING PLAN, \#2 WASTE TRANSFER LINES

PIPING PLAN, \#3

PIPING PLAN, \#4

PIPING PLAN, \#5

PIPING PLAN, \#6

PIPING PLAN, 241-AP VALVE PIT

PIPING PLAN, TANK 101

PIPING PLAN, TANK 102

PIPING PLAN, TANK 103

PIPING PLAN, TANK 104

PIPING PLAN, TANK 105

PIPING PLAN, TANK 106

PIPING PLAN, TANK 107

PIPING PLAN, TANK 108

PIPING PLAN, CENTRAL PUMP PIT, 241-AP-01A THRU 08A

PIPING SECTION \& DETAILS, CENTRAL PUMP PIT, 241-AP-01A

THRU 08A

PIPING PLAN, ANNULUS PUMP PIT, 241-AP-01B THRU 08B

PIPING SECTION \& DETAIL, ANNULUS PUMP PIT, 241-AP-O1B

THRU O8B

PIPING PLAN, LEAK DETECTION PIT, 241-AP-03C \& 05C

PIPING SECTION \& DETAILS, LEAK DETECTION PIT, 241-AP-03C \& $05 \mathrm{C}$

PIPING PLAN \& DETAILS, FLUSH PIT

PIPING PLAN \& DETAILS, SERVICE BLDG. 


\section{1-ER DIVERSION BOXES \& CATCH TANKS}

$\mathrm{H}-2-71614 / 1$

ELECTRICAL/INSTRUMENTATION DETAILS

\section{4-A LIFT STATION}

$\mathrm{H}-2-38214 / 1$

$\mathrm{H}-2-38215 / 1$

$\mathrm{H}-2-38218 / 1$

$\mathrm{H}-2-38220 / 1$

$\mathrm{H}-2-38222 / 1$

H-2-95298/1-2

\section{1-AW TANK FARM}

$\mathrm{H}-2-70339 / 1-2$

$\mathrm{H}-2-70340 / 1$

$\mathrm{H}-2-70352 / 1$

$\mathrm{H}-2-70367 / 1$

$\mathrm{H}-2-70387 / 1$

$\mathrm{H}-2-70388 / 1$

$\mathrm{H}-2-70389 / 1$

$\mathrm{H}-2-70390 / 1$

$\mathrm{H}-2-70391 / 1$

$\mathrm{H}-2-70393 / 1$

H-2-70398/1-2

$\mathrm{H}-2-70399 / 1$

$\mathrm{H}-2-70400 / 1$

$\mathrm{H}-2-7040 \mathrm{l} / \mathrm{l}$

$\mathrm{H}-2-70403 / 1$

$\mathrm{H}-2-70404 / 1$

$\mathrm{H}-2-70405 / 1$

$\mathrm{H}-2-70406 / 1$

$\mathrm{H}-2-70407 / 1$

$\mathrm{H}-2-70408 / 1$

H-2-70411/1-2

$\mathrm{H}-2-70412 / 1$

$\mathrm{H}-2-70413 / 1-2$

$\mathrm{H}-2-70414 / 1$

$\mathrm{H}-2-70415 / 1$

$\mathrm{H}-2-70416 / 1-2$

$\mathrm{H}-2-70417 / 1-2$

$\mathrm{H}-2-74896 / 1$

$\mathrm{H}-2-74896 / 3$

$\mathrm{H}-2-77118 / 1$

$\mathrm{H}-2-90275 / 1$

$\mathrm{H}-2-90392 / 1$

$\mathrm{H}-2-90906 / 1$

$\mathrm{H}-2-90909 / 1$

$\mathrm{H}-2-90928 / 1$

$\mathrm{H}-2-90929 / 1$
VENTILATION AIR FLOW \& CONTROL DIAGRAM \& SCHEDULES

VENTILATION PLANS \& DETAILS

INSTRUMENTATION DETAILS

INSTR. SERVICE PIT RAD MONITORING ARRGT. \& MISC. DETAILS

EFD, WASTE TRANSFER SYSTEM

296-A-25 STACK MONITOR INSTALLATION, WIRING DIAGRAM

HVAC K2 SYSTEM EQUIPMENT PLANS, SECTIONS \& DETAILS HVAC K2 SYSTEM EQUIPMENT SCHEDULES

INSTRUMENTATION, TUBE ROUTING \& TRANSMITTER ENCLOSURE

INSTRUMENTATION, FLOW \& RADIATION MONITORING SYSTEM

EFD, 241-AW

EFD, TANK 241-AW-101

EFD, TANK 241-AW-102

EFD, TANK 241-AW-103

EFD, TANK 241-AW-104

EFD, TANK 241-AW-106

PIPING PLAN, 241-AW TANK FARM TO 242-A BLDG.

PIPING PLAN, 241-AW TANK FARM

PIPING PLAN, 241-AW TANK FARM

PIPING PLAN, VALVE PITS 241-AW-A \& B

PIPING PLAN, TANK 101

PIPING PLAN, TANK 102

PIPING PLAN, TANK 103

PIPING PLAN, TNAK 104

PIPING PLAN, TANK 105

PIPING PLAN, TANK 106

PIPING PLAN, CENTRAL PUMP PIT 241-AW-01A THRU 06A

PIPING PLAN \& DETAILS, ANNULUS PUMP PIT 241-AW-01B-THRU 06B

PIPING PLAN \& DETAILS, LEAK DETECTION PIT 241-AW-01C THRU 06C

PIPING PLAN \& DETAILS, DRAIN PIT 241-AW-02D

PIPING PLAN \& DETAILS, FEED PUMP PIT 241-AW-02E

PIPING PLAN \& DETAILS, FLUSH PIT 241-AW

PIPING PLAN \& SECTIONS, SERVICE PIT

296-A-27 STACK MONITOR INSTALLATION

296-A-27 STACK MONITOR DIAGRAM

ELECTRICAL ELEMENTARY, EFD

ELECTRICAL/INSTRUMENT, EFD

EFD, TANK 241-AW-105

HVAC K1 SYSTEM EQUIPMENT PLANS \& ELEVATIONS

HVAC K1 SYSTEM EQUIPMENT SCHEDULES \& NOTES

ELECTRICAL ELEMENTARY DIAGRAM

ELECTRICAL CONNECTION DIAGRAM 
241-AY, 241-AZ \& MISCELLANEOUS A-FARM FACILITY SUPPORT DRAKINGS 241-AZ

$\mathrm{H}-2-38218 / 2$
$\mathrm{H}-2-67283 / 1$
$\mathrm{H}-2-67307 / 1$
$\mathrm{H}-2-68337 / 1$
$\mathrm{H}-2-68338 / 1-3$
$\mathrm{H}-2-68340 / 1$
$\mathrm{H}-2-68409 / 1$
$\mathrm{H}-2-72187 / 2$
$\mathrm{H}-2-72187 / 1$
$\mathrm{H}-2-92970 / 1$
$\mathrm{H}-2-94856 / 1$
$\mathrm{H}-2-94858 / 1$
$\mathrm{H}-2-94858 / 2$
$\mathrm{H}-2-95413 / 1$
$\mathrm{H}-2-95413 / 3$
$\mathrm{H}-2-95413 / 2$
INSTRUMENTATION, INSTR. ENCL. 244-A BLDG., ARRGT. \& DETAILS VENTILATION AIR FLOW DIAGRAM, ANNULUS

INSTR., ANNULUS VENT RADIATION MONITOR SYSTEM, ARRANGEMENT \& WIRING

INSTR., TEMP. STR. GA \& LK DET. ELEMENTS TERMINAL BOX INSTR. INSTR., TUBE ROUTING \& TRANSMITTER ENCLOSURE, ARRANGEMENT \& DETAILS

INSTR., REAR PANEL WIRING \& ELEMENTARY DIAGRAM INSTR., REAR PANEL WIRING \& ELEMENTARY DIAGRAM

INSTR., 101-102 TANK SLUDGE LEVEL, LIQUID LEVEL ALARM, ASSEMBLY \& DETAILS

LIQUID LEVEL ALARM, ASSEMBLY \& DETAIL

RADIATION MONITORING, ANNULUS VAPOR SAMPLING

SLUDGE MEASUREMENT, 6" RISER CAP \& SLUDGE FLOAT

RADIATION DETECTORS \& LTG. CHECK VALVE CAISSON

RADIATION DETECTORS \& LTG. CHECK VALVE CAISSON

INSTR., LIQUID LEVEL INDICATION \& ALARM MOD. ASSY. \& DETAILS INSTR., LIQUID LEVEL INDICATION \& ALARM MOD. INTERCONNECTION DIAGRAM

INSTR., LIQUID LEVEL INDICATION \& ALARM MOD. ASSY. \& DETAILS

241-AY, 241-AZ \& MISCELLANEOUS A-FARM FACILITY SUPPORT DRAWINGS

A-FARM MISCELLANEOUS: (241-A-271, A-401, A-702, A-701, A-08, A-151, TK-A-417, A-A VALVE PIT, A-B VALVE PIT, AX-A VALVE PIT \& AX-B VALVE PIT)

$\mathrm{H}-2-33681 / 1-2$

$\mathrm{H}-2-38375 / 1$

$\mathrm{H}-2-38935 / 1$

$\mathrm{H}-2-46789 / 2$

$\mathrm{H}-2-55135 / 1$

$H-2-56039 / 1$

$\mathrm{H}-2-56139 / 1$

$\mathrm{H}-2-56142 / 1-2$

$\mathrm{H}-2-56349 / 1$

$\mathrm{H}-2-56812 / 2$

$\mathrm{H}-2-56813 / 1$

$\mathrm{H}-2-56824 / 1$

$\mathrm{H}-2-56825 / 1$

$\mathrm{H}-2-57940 / 1$

$\mathrm{H}-2-62893 / 1$

$\mathrm{H}-2-62896 / 2$

$\mathrm{H}-2-62896 / 3$

$\mathrm{H}-2-62896 / 1$
INSTRUMENT PANEL ELEVATION, 241-A-271 BLDG.

ELECTRICAL ELEMENTARY DIAGRAMS

ELECTRICAL PLANS \& ELEVATIONS, PANELBOARD SCHEDULES

ELEC. / INSTR., PLANS, ELEV., DETAILS \& ELEMENTARY DIAGRAMS INSTRUMENTATION, SCHEM. 7 DETAILS, 241-A-151 DIVERSION BOX, OUTSIDE COOL

CONTROL HOUSE, INSTRUMENT PANEL ARRANGEMENT

CONTACT CONDENSER INSTALL., INSTR. PANEL ARRGT. \& PIPING, CONTROL HOUSE

INSTRUMENT ENGINEERING FLOW DIAGRAM

241-A-701 \& 241-A-271, COMPRESSOR \& CONTROL BLDG., WIRING DIAGRAMS

ELECTRICAL POWER LIGHTING \& CONNECTION DIAGRAM

ELECTRICAL ELEMENTARY \& CONNECTION DIAGRAM

INSTRUMENTATION, PANEL ARRANGEMENT, PIPING \& WIRING

INSTRUMENTATION, RACK ARRGT., ADDTNL FAC. FOR PUREX TK. FARM CONDENSER COOLING TOWER, SUMPS, PIPING, SCHEMATIC DIAGRAM MCC-A5 \& 241-A-702, FILTER BLDG. PLAN, DIAGRAMS \& DETAILS INSTR., ARRGT. PLANS, PANEL ELEVATION \& CONNECTION DIAGRAM INSTR., IMPULSE \& SAMPLE PIPING, VENT SYSTEM EXPANSION, 241-A-702 \& 241-A-271 BUILDINGS

INSTR., IMPULSE \& SAMPLE PIPING, VENT SYSTEM EXP., 241-A-702 \& 241-A-271 BLDGS. 
241-AY, 241-AZ \& MISCELLANEOUS A-FARM FACILITY SUPPORT DRAWINGS

A-FARM MISCELLANEOUS: (241-A-271, A-401, A-702, A-701, A-08, A-151, TK-A-417, (CON'T)

$\mathrm{H}-2-64775 / 1$

$\mathrm{H}-2-68341 / 1$

$\mathrm{H}-2-69163 / 1$

$\mathrm{H}-2-69171 / 1$ $\mathrm{H}-2-70760 / 1$ $\mathrm{H}-2-71781 / 1$ $\mathrm{H}-2-71781 / 2$ $\mathrm{H}-2-73732 / 1$ $\mathrm{H}-2-73732 / 2$ $\mathrm{H}-2-73732 / 3$ $\mathrm{H}-2-73740 / 1$ $\mathrm{H}-2-75960 / 1-2$ $\mathrm{H}-2-90936 / 2$ $\mathrm{H}-2-90937 / 1-2$ $\mathrm{H}-2-91092 / 1$ $\mathrm{H}-2-91114 / 1$ $\mathrm{H}-2-92516 / 2$ $\mathrm{H}-2-92516 / 3$ $\mathrm{H}-2-93119 / 1$ $\mathrm{H}-2-93119 / 2$ $\mathrm{H}-2-93206 / 3$ $\mathrm{H}-2-93206 / 1$ $\mathrm{H}-2-99388 / 3$ $\mathrm{H}-2-99388 / 1$ $\mathrm{H}-2-99388 / 2$

\section{1-AY TANK FARM}

$\mathrm{H}-2-64365 / 1$

$\mathrm{H}-2-64365 / 2$

$\mathrm{H}-2-64365 / 3$

$\mathrm{H}-2-64367 / 1-2$

$\mathrm{H}-2-64371 / 1$

$\mathrm{H}-2-64377 / 1$

$\mathrm{H}-2-64461 / 1$

$\mathrm{H}-2-64462 / 1$

$\mathrm{H}-2-93375 / 2$

$\mathrm{H}-2-93375 / 3$

$\mathrm{H}-2-93375 / 4$

$\mathrm{H}-2-93375 / 5$

$\mathrm{H}-2-93375 / 6$

$\mathrm{H}-2-99388 / 1$

$\mathrm{H}-2-99388 / 2$

$\mathrm{H}-2-99388 / 3$
$A-A$ VALVE PIT, A-B VALVE PIT, $A X-A$ VALVE PIT \& AX-B VALVE PIT)

INSTR. , ARRGT. PLANS, PANEL ELEV. \& CONNECTION DIAGRAM INSTRUMENTATION, CONTROL HOUSE PANEL ARRGT. \& WIRING ELECTRICAL, DATA AQUISITION SYSTEM PLAN, HEAT TRACE DETAILS, PANEL SCHEDULES \& ONE-LINE DIAGRAM INSTR., TEMP. MONITOR SYSTEM, INTERCONNECTION DIAGRAM, 241-A ENG. FLOW DIAGRAM, VALVE PITS TO 241-AX, AY \& AZ TANK FARMS ELECTRICAL, SUBSTATION "A" ARRGT. \& CONNECTION DIAGRAMS ELECTRICAL, SUBSTATION "A" ARRGT. \& CONNECTION DIAGRAMS INSTRUMENTATION, INSTR. PANEL \& RACK ARRGT. \& WIRING INSTRUMENTATION, INSTR. PANEL \& RACK ARRGT. \& WIRING INSTRUMENTATION, INSTR. PANEL \& RACK ARRGT. \& WIRING ELECTRICAL, ANNUNCIATOR, ELEMENTARY DIAGRAM

ELEC./INSTR. PLANS, ELEVATION \& DIAGRAMS ELEC./INSTR. PLOT PLAN, PLAN \& PANEL ARRANGEMENT ELEC./INSTR. PLAN, EL., DETAIL \& WIRE RUN LIST JUMPER ARRGT., HEAT TRACING, VALVE PIT, 241-AX-A \& 241-AX-B ELECTRICAL PLANS \& DETAILS, A \& AX TANK FARMS FILTER BLDG., STACK MONITOR INSTR., 296-A-17 FILTER BLDG., STACK MONITOR INSTR., 296-A-17 A-417, TANK WEIGHT FACTOR, MODIFICATION A-417, TANK WEIGHT FACTOR, MODIFICATION ELECTRICAL, VALVE PIT, 241-A-A JUMPER VALVE POSITIONS GRAPHIC PANEL LAYOUT \& PLOT PLAN, VALVE PIT, 241-A JUMPER EQUIPMENT LOCATION NAMEPLATE SCHEDULE

ELECTRICAL, EQUIPMENT LOCATIONS, TANK FARMS 241-A, AX \& AY EQUIPMENT LOCATION NAMEPLATE SCHEDULE

(INCLUDES 244-AR-151)

241-AY-801, INSTRUMENT PANEL ARRANGEMENT

241-AY-801, INSTRUMENT PANEL ARRANGEMENT, REAR PANEL WIRING INSTRUMENT PANEL ARRANGEMENT, REAR PANEL PIPING

INSTR., TUBE ROUTINE \& TRANSMITTER INCL., ARRGT. \& DETAILS 241-AY, PANEL IN 241-A-271 CONTROL HOUSE, ARRGT. \& WIRING INSTR., TANK SLUDGE LIQUID LEVEL ALARM, ASSY. \& DETAILS VENTILATION, AIR FLOW DIAGRAM

VENTILATION, EQUIPMENT SCHEDULE \& CONTROL DIAGRAM

TEMPERATURE ELEMENT SCANNING, INSTRUMENTATION BLOCK DIAGRAM TEMPERATURE ELEMENT SCANNING, INSTRUMENTATION BLOCK DIAGRAM TEMPERATURE ELEMENT SCANNING, INSTRUMENTATION BLOCK DIAGRAM TEMPERATURE ELEMENT SCANNING, INSTRUMENTATION BLOCK DIAGRAM TEMPERATURE ELEMENT SCANNING, INSTRUMENTATION BLOCK DIAGRAM ELECTRICAL, EQUIPMENT LOCATIONS, TANK FARMS 24I-A, AX \& AY EQUIPMENT LOCATION NAMEPLATE SCHEDULE EQUIPMENT LOCATION NAMEPLATE SCHEDULE 
241-AN TANK FARM

\section{H-2-71116/1-2}

$\mathrm{H}-2-71117 / 1$

$\mathrm{H}-2-71925 / 1-5$

$\mathrm{H}-2-71926 / 1$

$\mathrm{H}-2-71929 / 1-2$

$\mathrm{H}-2-71930 / 1-2$

$\mathrm{H}-2-71932 / 1$

$\mathrm{H}-2-71936 / 1-3$

$\mathrm{H}-2-71937 / 1$

$\mathrm{H}-2-71956 / 1$

$\mathrm{H}-2-71957 / 1-2$

$\mathrm{H}-2-71958 / 1$

$\mathrm{H}-2-71960 / 1$

$\mathrm{H}-2-71961 / 1$

$\mathrm{H}-2-71964 / 1-2$

$\mathrm{H}-2-71966 / 1$

$\mathrm{H}-2-71986 / 1$

$\mathrm{H}-2-71987 / 1-2$

$\mathrm{H}-2-71989 / 1$

$\mathrm{H}-2-71990 / 1$

$\mathrm{H}-2-71991 / 1$

$\mathrm{H}-2-71992 / 1$

$\mathrm{H}-2-71993 / 1$

$\mathrm{H}-2-71994 / 1$

$\mathrm{H}-2-71995 / 1-2$

$\mathrm{H}-2-71996 / 1$

$\mathrm{H}-2-71997 / 1-2$

$\mathrm{H}-2-71998 / 1$

$\mathrm{H}-2-71999 / 1-2$

$\mathrm{H}-2-72001 / 1-2$

$\mathrm{H}-2-72038 / 1$

$\mathrm{H}-2-72039 / 1$

$\mathrm{H}-2-73843 / 1$

$\mathrm{H}-2-73848 / 1$

$\mathrm{H}-2-76608 / 1$

242-A EVAPORATOR

H-2-92505/1-2

\section{2-T EVAPORATOR}

$\mathrm{H}-2-27282 / 1$

$\mathrm{H}-2-35878 / 1$

$\mathrm{H}-2-36873 / 1$

$\mathrm{H}-2-38738 / 1$

$\mathrm{H}-2-73824 / 3-5$

$\mathrm{H}-2-93434 / 1-2$

$\mathrm{H}-2-97493 / 1$
ELEC. POWER, INSTR, , HEAT TRACE \& LEAK DETECTION PLANS

ELECTRICAL, MISC. PLANS \& DETAILS

ELECTRICAL POWER \& CONTROL PLANS \& DETAILS

ELECTRICAL DETAILS

ELECTRICAL HEAT TRACE ELEMENTARY DIAGRAM \& CONTROL PANEL

ELECTRICAL, LEAK DETECTION PLANS \& DETAILS

ELECTRICAL INSTRUMENT PLANS

HVAC, EQUIPMENT PLAN, ELEVATION \& DETAILS

HVAC, EQUIPMENT SCHEDULES, DETAILS \& GENERAL NOTES

INSTRUMENT PANEL ARRANGEMENT

INSTRUMENT PANEL ARRANGEMENT

INSTRUMENT REAR PANEL PIPING

INSTRUMENT REAR PANEL WIRING

INSTRUMENT REAR PANEL WIRING

INSTRUMENT PANEL DETAILS, TANK 241-AN-107

INSTRUMENT SWITCH RACK ARRANGEMENT \& DETAILS

PIPING PLAN, 241-AN TANK FARM

PIPING PLAN, 241-AN TANK FARM

PIPING PLAN, VALVE PITS, 241-AN/A \& B

SECTION \& DETAILS, VALVE PITS, 241-AN/A \& B

PIPING PLAN, TANK 101

PIPING PLAN, TANK 102

PIPING PLAN, TANK 103

PIPING PLAN, TANK 104

PIPING PLAN, TANK 105

PIPING PLAN, TANK 106

PIPING PLAN \& DETAILS, LEAK DETECTION PIT, 0IC-07C

PIPING PLAN, CENTRAL PUMP PIT, 01A-17A

PIPING PLAN \& DETAILS, ANNULUS PUMP PIT, 01B-07B

PIPING PLAN \& DETAILS, FLUSH PIT, 241-AN

PIPING PLAN, 241-AN TANK FARM

PIPING PLAN, TANK 107

PIPING PLAN, 241-AX/241-AN SYSTEM \#5

PIPING PLAN, 241-AN-01D \& 01E

PIPING PLAN, SECTION \& DETAILS, 241-AN SERVICE PIT MOD.

296-A-22 VESSEL VENT STACK MONITOR INSTALLATION, DETAILS

ELECTRICAL ONE-LINE \& CONTROL DIAGRAM

ELECTRICAL ONE-LINE DIAGRAM \& ELEMENTARY DIAGRAM

ELECTRICAL, FIRE ALARM SYSTEM

VENTILATION, FLOW DIAGRAM, 242-T

ELECTRICAL, PWR, INSTR., HEAT TRACE DISTR. PLAN, SYS. NO. 1 296-T-17 BUILDING, EXHAUST STACK MONITOR, FLOW DIAGRAMS

ELECTRICAL, RADIO FIRE ALARM PLAN \& DIAGRAM 
242-S EVAPORATOR

\author{
H-2-46323/1 \\ $\mathrm{H}-2-46330 / 1$ \\ $\mathrm{H}-2-46361 / 1$ \\ $\mathrm{H}-2-46362 / 1$ \\ $\mathrm{H}-2-4642 \mathrm{l} / \mathrm{l}-3$ \\ $\mathrm{H}-2-46422 / 1-3$ \\ $\mathrm{H}-2-46423 / 1$ \\ $\mathrm{H}-2-46427 / 1$ \\ $\mathrm{H}-2-46431 / 1$ \\ $\mathrm{H}-2-46432 / 1$ \\ $\mathrm{H}-2-46437 / 1$ \\ $\mathrm{H}-2-46438 / 1$ \\ $\mathrm{H}-2-46785 / 2-3$ \\ $\mathrm{H}-2-46789 / 2$ \\ $\mathrm{H}-2-71784 / 1$
}

\section{4-AR VAULT}

$\mathrm{H}-2-34632 / 1$

$\mathrm{H}-2-34632 / 2$

$\mathrm{H}-2-34703 / 1$

$\mathrm{H}-2-34797 / 1$

$\mathrm{H}-2-35081 / 3$

$\mathrm{H}-2-37865 / 1$

$\mathrm{H}-2-38035 / 1$

$\mathrm{H}-2-38035 / 2$

$\mathrm{H}-2-38218 / 2$

$\mathrm{H}-2-58750 / 1$

$\mathrm{H}-2-62012 / 1$

$\mathrm{H}-2-62018 / 1$

$\mathrm{H}-2-62018 / 2$

$\mathrm{H}-2-62018 / 3$

$\mathrm{H}-2-62019 / 1$

$\mathrm{H}-2-62028 / 1$

$\mathrm{H}-2-62039 / 1$

$\mathrm{H}-2-62055 / 1$

$\mathrm{H}-2-62056 / 1$

$\mathrm{H}-2-62058 / 1$

$\mathrm{H}-2-63087 / 1$

$\mathrm{H}-2-63902 / 1$

$\mathrm{H}-2-63905 / 1$

$\mathrm{H}-2-63932 / 1$

$\mathrm{H}-2-63943 / 1$

$\mathrm{H}-2-70137 / 3$

$\mathrm{H}-2-70137 / 4$

$\mathrm{H}-2-72187 / 2$

$\mathrm{H}-2-72493 / 1$

$\mathrm{H}-2-72493 / 2-3$

$\mathrm{H}-2-76719 / 1$
ENGINEERING FLOW DIAGRAM, CONDENSER ROOM

ENGINEERING FLOW DIAGRAM, AIR SAMPLING

VESSEL VENT SYSTEM, ARRANGEMENT

VESSEL VENT SYSTEM, DETAIL

FIRE PROTECTION, SPRINKLER SYSTEM, 1ST \& 2ND FLOOR PLANS

FIRE PROTECTION, SPRINKLER SYSTEM PLANS, SECTIONS \& DETAILS

FIRE PROTECTION, SPRAY SYSTEM PLANS, SECTIONS \& DETAILS

ENGINEERING FLOW DIAGRAM, CONTROL \& AMU ROOMS

ELECTRICAL, FIRE ALARM \& COMMUNICATION PLANS

ELECTRICAL, FIRE ALARM \& COMMUNICATION DIAGRAMS

ELECTRICAL, PROCESS CONTROL DIAGRAM

ELECTRICAL, PROCESS CONTROL DIAGRAM

INSTRUMENTATION, EXHAUST UNIT, ENCLOSURE ASSEMBLYS

ELEC./INSTR., PLANS, ELEV., DETAILS \& ELEMENTARY DIAGRAMS

ELECTRICAL, SUBSTATION "S" ARRANGEMENT \& CONN. DIAGRAMS

BRIDGE CRANE, ELEMENTARY DIAGRAM

BRIDGE CRANE, ELEMENTARY DIAGRAM

HVAC, CONTROL DIAGRAM

EMERGENCY DIESEL ENGINE, STARTING CONTROL CIRCUITS

OIL-FREE COMPRESSOR, ELECTRICAL DETAIL

ELECTRICAL PLANS \& DETAILS, 244-AR VAULT

FIRE ALARM, SCHEMATIC DIAGRAM

FIRE ALARM, SCHEMATIC DIAGRAM

INSTRUMENTATION, INSTR. ENCL. 244-A BLDG., ARRGT. \& DETAILS ARRANGEMENT, ONE-LINE DIAGRAM, 244-AR VAULT

ELECTRICAL DIAGRAM

480-VOLT, SWITCHGEAR MODIFICATIONS

480-VOLT, SWITCHGEAR MODIFICATIONS, UNIT 12

480-VOLT, SWITCHGEAR MODIFICATIONS, UNIT 11

ONE-LINE DIAGRAM, SWITCHGEAR \& MCC ARRANGEMENTS

HVAC, AIR FLOW DIAGRAM

INSTRUMENT CONNECTION \& ELEMENTARY DIAGRAMS

CELL \#1 - EQUIPMENT ARRANGEMENT

CELL \#2 - EQUIPMENT ARRANGEMENT

CELL \#3 - EQUIPMENT ARRANGEMENT

COOLING, H2O DIVERSION BOX VALVE, CENTRAL ELEC. PLAN \& DIAG.

ELECTRICAL ONE-LINE \& ELEMENTARY DIAGRAMS

EFD \& PIPING PLAN, DIESEL FUEL SYSTEM

ELECTRICAL DIAGRAMS \& DETAILS

CELL \#3 - ARRANGEMENT, 244-AR VAULT

PROCESS ALARM PANEL, MODIFICATIONS DIAGRAM

PROCESS ALARM PANEL, MODIFICATIONS DIAGRAM

INSTR., 101 \& 102 TANK SLUDGE LEVEL, LIQUID LEVEL ALARM, ASSEMBLY \& DETAILS

FILTER ASSEMBLY, VESSEL VENT SYSTEM

FILTER DETAILS, VESSEL VENT SYSTEM

INSTRUMENT, INTERCONNECTION DIAGRAM 


\section{4-AR VAULT (CON'T)}

$\mathrm{H}-2-76719 / 2$

$\mathrm{H}-2-76719 / 3$

$\mathrm{H}-2-76719 / 4$

$\mathrm{H}-2-76728 / 1$

$\mathrm{H}-2-76729 / 1$

$\mathrm{H}-2-76730 / 1$

$\mathrm{H}-2-76730 / 2$

$\mathrm{H}-2-76730 / 3$

$\mathrm{H}-2-76730 / 4$

$\mathrm{H}-2-77161 / 1$

$\mathrm{H}-2-77163 / 3$

$\mathrm{H}-2-77168 / 1$

$\mathrm{H}-2-77168 / 2$

$\mathrm{H}-2-77238 / 1$

$\mathrm{H}-2-91006 / 1$

$\mathrm{H}-2-91007 / 1$

$\mathrm{H}-2-91010 / 1$

$\mathrm{H}-2-91025 / 1$

$\mathrm{H}-2-91191 / 1-2$

$\mathrm{H}-2-93994 / 1$

$\mathrm{H}-2-93994 / 2$

$\mathrm{H}-2-93995 / 1$

$\mathrm{H}-2-93996 / 1$

$\mathrm{H}-2-93997 / 1$

$\mathrm{H}-2-93998 / 1$

$\mathrm{H}-2-94009 / 1$

$\mathrm{H}-2-94009 / 2$

$\mathrm{H}-2-94009 / 3$

$\mathrm{H}-2-94013 / 1$

$\mathrm{H}-2-94013 / 2$

$\mathrm{H}-2-94013 / 3$

$\mathrm{H}-2-94013 / 4$

$\mathrm{H}-2-94014 / 1$

H-2-97520/1
INSTRUMENT, INTERCONNECTION DIAGRAM

INSTRUMENT, INTERCONNECTION DIAGRAM

INSTRUMENT, INTERCONNECTION DIAGRAM

ELECTRICAL PLANS \& DETAILS

ELECTRICAL ONE-LINE DIAGRAM \& DETAILS

ELECTRICAL ELEMENTARY DIAGRAM

ELECTRICAL ELEMENTARY. DIAGRAM

ELECTRICAL ELEMENTARY DIAGRAM

ELECTRICAL ELEMENTARY DIAGRAM

ELECTRICAL ONE-LINE DIAGRAM

ELECTRICAL ELEMENTARY WIRING DIAGRAM

INSTRUMENT, INTERCONNECTION DIAGRAM

INSTRUMENT, INTERCONNECTION DIAGRAM

ELECTRICAL ONE-LINE DIAGRAM

HVAC, AIRFLOW \& CONTROL DIAGRAM, SYSTEM DESCRIPTION

HVAC, PLANS, DETAILS \& DIAGRAMS

ELECTRICAL DIAGRAM

MONITOR RACK, ELECTRICAL DIAGRAM

296-A-13 STACK MONITOR ASSEMBLY

HVAC, PLAN \& SECTIONS

HVAC, PLAN \& SECTIONS

HVAC, SECTIONS \& DETAILS

HVAC, SECTIONS \& DETAILS

HVAC, SECTIONS \& DETAILS

HVAC, SECTIONS \& DETAILS

INSTRUMENTATION, PIPING \& INSTRUMENT DIAGRAM

INSTRUMENTATION, PIPING \& INSTRUMENT DIAGRAM

INSTRUMENTATION, PIPING \& INSTRUMENT DIAGRAM

ELECTRICAL, ELEMENTARY \& CONNECTION DIAGRAM, 291-AR

ELECTRICAL, ELEMENTARY \& CONNECTION DIAGRAM, 291-AR

ELECTRICAL, ELEMENTARY \& CONNECTION DIAGRAM, 291-AR

ELECTRICAL, ELEMENTARY \& CONNECTION DIAGRAM, 291-AR

ELECTRICAL PLANS \& ELEVATIONS

ELECTRICAL, RADIO FIRE ALARM PLAN \& DIAGRAM

\section{4-AR}

$\mathrm{H}-2-70695 / 3$

ELECTRICAL DETAILS AND POLE LINE PLAN \& DETAILS

$\mathrm{H}-2-99070 / 1$

ELEC./CIVIL RELOCATION PLAN, DETAILS \& DIAGRAM

\section{COMPUTER AUTOMATED SURVEILLANCE SYSTEM (CASS)}

$\mathrm{H}-2-38935 / 1$

ELECTRICAL PLANS \& ELEVATIONS, PANELBOARD SCHEDULES 